\title{
Search for low-mass leptoquarks using the llqq final states in $\mathrm{Pb}-\mathrm{Pb}$ ultra-peripheral collisions
}

\author{
Li-Gang Xia \\ School of Physics, Nanjing University, \\ No. 22 Hankou Road, Nanjing, China \\ E-mail: ligang.xia@cern.ch
}

ABSTRACT: After a review over past experiments and theoretical requirements from lowenergy flavour physics, we argue that the possibility of low-mass leptoquarks (LQ) cannot be fully excluded due to the assumptions made in the measurements. Therefore we propose to search for the pair production of low-mass leptoquarks in $\mathrm{Pb}-\mathrm{Pb}$ ultra-peripheral collisions with the least model dependence (assuming a small coupling constant $\lesssim 0.1$ ). There are a couple of advantages: 1) high photon flux provides high production rate for low mass LQs; 2) the background contamination is much lower than that in $p-p$ collisions. The analysis strategy permits a leptoquark to decay to all possible lepton-plus-quark modes. Taking the scalar LQ, $S_{3}$, with an electric charge $|q|=\frac{4}{3} e$, as example, the mass point of $100 \mathrm{GeV}$ can be excluded at the $95 \%$ confidence level using a dataset of $4 \mathrm{pb}^{-1} \mathrm{~Pb}-\mathrm{Pb}$ ultra-peripheral collisions at $\sqrt{s}=5.02 \mathrm{TeV}$ and the performance of the ATLAS detector in Run 2. The proposed method also applies to searching for high-mass LQs in $p$ - $p$ collisions as long as the LQ pair production mechanism dominates.

KEywords: Beyond Standard Model, Particle and resonance production, Exotics, Heavy Ion Experiments

ArXiv ePrint: 2012.15618 


\section{Contents}

1 Introduction 1

2 The bounds from the experimental searches and low-energy flavour physics

2.1 A robust lower mass bound from the OPAL experiments 4

2.2 The bounds from low-energy flavour physics 5

3 A model-independent searching method using the LQ pair production 6

4 Cross sections and MC simulation $\quad 6$

4.1 Cross sections in UPC 6

$\begin{array}{ll}4.2 \text { MC sample preparation } & 7\end{array}$

$\begin{array}{llr}5 & \text { Event selection and observable reconstruction } & 8\end{array}$

$\begin{array}{lll}6 & \text { Systematical uncertainties } & 9\end{array}$

7 Statistical interpretation and sensitivity results 13

8 Can we exclude a $100 \mathrm{GeV}$ LQ unambiguously? 14

$\begin{array}{lll}9 & \text { Summary } & 19\end{array}$

A Signal purity comparison between in $\mathrm{Pb}-\mathrm{Pb}$ collisions and in $p-p$ colli-

$\begin{array}{ll}\text { sions } & 19\end{array}$

\section{Introduction}

Leptoquarks (LQ) are bosons to connect the quark and lepton sectors. They carry fractional electric charge and color. LQs are predicted in many extensions of the Standard Model (SM), such as grand unified theories [1-4], technicolor models [5-9], composite LQ models [10-12] and other models $[13,14]$. They are able to explain the evidences of the lepton universality violation (LUV) measured in the $B$-meson decays [15-23]. Most recently, the LHCb experiment reported another evidence for LUV in the decays $B^{+} \rightarrow K^{+} l^{+} l^{-}$ with a significance of $3.1 \sigma$ [24]. Introducing one or more LQs seems a natural explanation $[25-31]$.

Various LQs have been searched for in the LEP, Tevatron, LHC and other experiments. Ref. [32] is a good review on the LQ physics and experimental searches up to 2016. Table 1 summarizes a list of experimental searches from different colliders. From the table, it seems that LQs are excluded up to $1 \mathrm{TeV}$ or more. However, all searching results are 


\begin{tabular}{|c|c|c|}
\hline Experiment & Exclusion region (condition) & Assumption \\
\hline $\mathrm{AMY}[36]$ & $4.3-26.3 \mathrm{GeV}\left(|q|=\frac{2}{3} e, B(\mathrm{LQ} \rightarrow e q)=1\right)$ & $\mathrm{SG}, \mathrm{LQ} \rightarrow e q / \mu q / \nu q(q=d, s)$ \\
\hline CELLO [37] & $7-20.5 \mathrm{GeV}$ & $\mathrm{LQ} \rightarrow \mu q / \nu q$ \\
\hline \multirow[t]{2}{*}{ ALEPH [38] } & $4-44 \mathrm{GeV}\left(|q|=\frac{1}{3} e\right)$ & $\mathrm{SG}, \mathrm{LQ} \rightarrow e u / \nu_{e} d$ \\
\hline & $6-44 \mathrm{GeV}\left(|q|=\frac{1}{3} e\right)$ & $\mathrm{SG}, \mathrm{LQ} \rightarrow \mu c / \nu_{\mu} s$ \\
\hline DELPHI [39] & $134 \mathrm{GeV}\left(B(\mathrm{LQ} \rightarrow e q)=1, \lambda=\sqrt{4 \pi \alpha_{\mathrm{em}}}\right)$ & $\mathrm{SG}, \mathrm{LQ} \rightarrow e q / \nu_{e} q(q=u, d)$ \\
\hline OPAL [40] & $\lambda<1$ for $200 \mathrm{GeV},\left(|q|=\frac{4}{3}, \mathrm{~B}(\mathrm{LQ} \rightarrow e q)=1\right)$ & $\mathrm{LQ} \rightarrow e q / \nu_{e} q,(q=u, d, s, c, b)$ \\
\hline \multirow[t]{2}{*}{ OPAL $[41]$} & $46.3 \mathrm{GeV}\left(|q|=\frac{4}{3}, B(\mathrm{LQ} \rightarrow e q / \mu q)=1\right)$ & $\mathrm{LQ} \rightarrow e q / \mu q,(q=u, d, s, c, b)$ \\
\hline & $45.5 \mathrm{GeV}\left(|q|=\frac{4}{3}, B(\mathrm{LQ} \rightarrow \tau q)=1\right)$ & $\mathrm{SG}, \mathrm{LQ} \rightarrow \tau q,(q=u, d, s, c, b)$ \\
\hline \multirow[t]{3}{*}{ OPAL [42] } & $50-100 \mathrm{GeV}\left(|q|=\frac{4}{3}, B(\mathrm{LQ} \rightarrow e q)=1\right)$ & $\mathrm{SL}, \mathrm{LQ} \rightarrow e q,(q=u, d, s, c, b)$ \\
\hline & $50-101 \mathrm{GeV}\left(|q|=\frac{4}{3}, B(\mathrm{LQ} \rightarrow \mu q)=1\right)$ & $\mathrm{SL}, \mathrm{LQ} \rightarrow \mu q,(q=u, d, s, c, b)$ \\
\hline & $50-99 \mathrm{GeV}\left(|q|=\frac{4}{3}, B(\mathrm{LQ} \rightarrow \tau q)=1\right)$ & $\mathrm{SL}, \mathrm{LQ} \rightarrow \tau q,(q=u, d, s, c, b)$ \\
\hline H1 [43] & $257 \mathrm{GeV}\left(B(\mathrm{LQ} \rightarrow e q)=1, \lambda=\sqrt{4 \pi \alpha_{\mathrm{em}}}\right)$ & $\mathrm{LQ} \rightarrow e q / \nu_{e} q(q=u, d)$ \\
\hline H1 [44] & $712 \mathrm{GeV}\left(\lambda=\sqrt{4 \pi \alpha_{\mathrm{em}}}\right)$ & $\mathrm{LQ} \rightarrow e q / \mu q,(q=u, d, s, c, b)$ \\
\hline H1 [45] & $800 \mathrm{GeV}\left(B(\mathrm{LQ} \rightarrow e q)=1, \lambda=\sqrt{4 \pi \alpha_{\mathrm{em}}}\right)$ & $\mathrm{LQ} \rightarrow e q / \nu_{e} q$ \\
\hline $\mathrm{CDF}[46]$ & $99 \mathrm{GeV}(\mathrm{B}(\mathrm{LQ} \rightarrow \tau b)=1)$ & $\mathrm{SG}, \mathrm{LQ} \rightarrow \tau b$ \\
\hline $\mathrm{CDF}[47]$ & $236 \mathrm{GeV}(\mathrm{B}(\mathrm{LQ} \rightarrow e q)=1)$ & $\mathrm{SG}, \mathrm{LQ} \rightarrow e q / \nu_{e} q(q=u, d)$ \\
\hline $\mathrm{CDF}[48]$ & $224 \mathrm{GeV}(\mathrm{B}(\mathrm{LQ} \rightarrow \mu q)=1)$ & $\mathrm{SG}, \mathrm{LQ} \rightarrow \mu q / \nu_{\mu} q(q=c, s)$ \\
\hline $\mathrm{CDF}[49]$ & $317 \mathrm{GeV}(\mathrm{B}($ Vector $\mathrm{LQ} \rightarrow \tau b)=1)$ & $\mathrm{SG}$, Vector $\mathrm{LQ} \rightarrow \tau b$ \\
\hline D0 [50] & $229 \mathrm{GeV}\left(\mathrm{B}\left(\mathrm{LQ} \rightarrow \nu_{\tau} b\right)=1\right)$ & $\mathrm{SG}, \mathrm{LQ} \rightarrow \nu_{\tau} b$ \\
\hline D0 [51] & $210 \mathrm{GeV}(\mathrm{B}(\mathrm{LQ} \rightarrow \tau b)=1)$ & $\mathrm{SG}, \mathrm{LQ} \rightarrow \tau b$ \\
\hline D0 $[52]$ & $316 \mathrm{GeV}(\mathrm{B}(\mathrm{LQ} \rightarrow \mu q)=1)$ & $\mathrm{SG}, \mathrm{LQ} \rightarrow \mu q / \nu_{\mu} q(q=c, s)$ \\
\hline D0 [53] & $295 \mathrm{GeV}(\mathrm{B}(\mathrm{LQ} \rightarrow e q)=1)$ & $\mathrm{SG}, \mathrm{LQ} \rightarrow e q / \nu_{e} q(q=u, d)$ \\
\hline D0 [54] & $247 \mathrm{GeV}\left(\mathrm{B}\left(\mathrm{LQ} \rightarrow \nu_{\tau} b\right)=1\right)$ & $\mathrm{SG}, \mathrm{LQ} \rightarrow \nu_{\tau} b$ \\
\hline ATLAS [55] & $200-534 \mathrm{GeV}(\mathrm{B}(\mathrm{LQ} \rightarrow \tau b)=1)$ & $\mathrm{SG}, \mathrm{LQ} \rightarrow \tau b$ \\
\hline ATLAS $[56]$ & $200-800 \mathrm{GeV}$ & $\mathrm{SG}, \mathrm{LQ} \rightarrow \nu_{\tau} t / \tau b / \nu_{\tau} b / \tau t$ \\
\hline \multirow[t]{2}{*}{ ATLAS [57] } & $0.4-1.8 \mathrm{TeV}(\mathrm{B}(\mathrm{LQ} \rightarrow e q)=1)$ & $\mathrm{SL}, \mathrm{LQ} \rightarrow e q(q=u, d, s, c, b)$ \\
\hline & $0.4-1.7 \mathrm{TeV}(\mathrm{B}(\mathrm{LQ} \rightarrow \mu q)=1)$ & $\mathrm{SL}, \mathrm{LQ} \rightarrow e q(q=u, d, s, c, b)$ \\
\hline \multirow[t]{2}{*}{ ATLAS [58] } & $0.9-1.48 \mathrm{TeV}(\mathrm{B}(\mathrm{LQ} \rightarrow e t)=1)$ & $\mathrm{SL}, \mathrm{LQ} \rightarrow e t$ \\
\hline & $0.9-1.47 \mathrm{TeV}(\mathrm{B}(\mathrm{LQ} \rightarrow \mu t)=1)$ & $\mathrm{SL}, \mathrm{LQ} \rightarrow \mu t$ \\
\hline ATLAS $[59]$ & $0.5-1.43 \mathrm{TeV}\left(|q|=\frac{1}{3}, \mathrm{~B}(\mathrm{LQ} \rightarrow \tau t)=1\right)$ & $\mathrm{SG}, \mathrm{LQ} \rightarrow \tau t / \nu_{\tau} b$ \\
\hline CMS [60] & $200-740 \mathrm{GeV}(\mathrm{B}(\mathrm{LQ} \rightarrow \tau b)=1)$ & $\mathrm{SG}, \mathrm{LQ} \rightarrow \tau b / \nu_{\tau} t$ \\
\hline \multirow[t]{2}{*}{ CMS $[61]$} & $200-1730 \mathrm{GeV}\left(\mathrm{B}\left(\mathrm{LQ} \rightarrow e^{-} u\right)=1, \lambda=1\right)$ & $\mathrm{SG}, \mathrm{LQ} \rightarrow e^{-} u$ \\
\hline & $300-660 \mathrm{GeV}\left(\mathrm{B}\left(\mathrm{LQ} \rightarrow \mu^{-} c\right)=1, \lambda=1\right)$ & $\mathrm{SG}, \mathrm{LQ} \rightarrow \mu^{-} c$ \\
\hline CMS [62] & $200-850 \mathrm{GeV}(\mathrm{B}(\mathrm{LQ} \rightarrow \tau b)=1)$ & $\mathrm{SG}, \mathrm{LQ} \rightarrow \tau b$ \\
\hline CMS $[63]$ & $200-740 \mathrm{GeV}(\mathrm{B}(\mathrm{LQ} \rightarrow \tau b)=1, \lambda=1)$ & $\mathrm{SG}, \mathrm{LQ} \rightarrow \tau^{+} b$ \\
\hline CMS $[64]$ & $900 \mathrm{GeV}\left(|q|=\frac{1}{3}, B(\mathrm{LQ} \rightarrow t \mu / t \tau / b \nu)=1\right)$ & $\mathrm{LQ} \rightarrow t \mu / t \tau / b \nu$ \\
\hline CMS [65] & $250-1020 \mathrm{GeV}(\mathrm{B}(\mathrm{LQ} \rightarrow \tau b)=1)$ & $\mathrm{SG}, \mathrm{LQ} \rightarrow \tau b$ \\
\hline CMS [35] & $950 \mathrm{GeV}\left(\mathrm{B}\left(\mathrm{LQ} \rightarrow \tau^{-} t\right)=\mathrm{B}\left(\mathrm{LQ} \rightarrow \nu_{\tau} b\right)=0.5\right)$ & $\mathrm{SG}, \mathrm{LQ} \rightarrow \tau^{-} t / \nu_{\tau} b$ \\
\hline
\end{tabular}

Table 1. Overview of the experimental searches for the leptoquarks. "SG" represents the assumption that a LQ couples to the same generation of fermions. "SL" represents the assumption that a LQ couples to single lepton and all possible quarks. It should be noted that the presented exclusion region may be just part of the experiment results. 
interpreted with assumptions. One common assumption is that a LQ couples only to the same generation of fermions (this assumption is denoted by "SG" in table 1). For example, a so-called "third-generation" LQ with a charge $+\frac{2}{3} e$ only couples to $b \tau^{+}$or $t \bar{\nu}_{\tau}$. This assumption is not appropriate because of more and more strong evidences for LUV. Taking the decay $B^{+} \rightarrow K^{+} l^{+} l^{-}$as in the LHCb measurement [24] as example, a LQ must be able to couple to $\mu / e$ and $b / s$ quark if LQ is the right solution. A much looser assumption is that a LQ couples to a single lepton plus all possible quarks (this assumption is denoted by "SL" in table 1). This assumption was used in the OPAL experiment [41, 42]. Inspired by their strategy, a model-independent method is proposed in section 3. For the measurements using single LQ production processes, we have to interpret the results as a function of the LQ mass and the coupling constant between a LQ and the fermions (denoted by $\lambda$ or $\lambda_{l q}$ for the lepton $l$ and the quark $q$ ). One common way is to set constraints on the LQ mass assuming a coupling strength which is equal to that in the electromagnetic interaction, namely, $\lambda=\sqrt{4 \pi \alpha_{\mathrm{em}}}$ in table 1 , where $\alpha_{\mathrm{em}} \approx \frac{1}{137}$ is the fine structure constant.

A combined fit to all measurements is desirable to avoid the assumptions, but not available for the moment. In section 2 , we will show that $80 \mathrm{GeV}$ is a robust lower limit for the LQ with an electric charge $|q|=\frac{4}{3}$ e based on the OPAL measurements [41, 42]. Considering a LQ with an electric charge $|q|=\frac{4}{3} e$, it has 9 decay modes (LQ $\rightarrow l+q$ with $l=e, \mu, \tau$ and $q=d, s, b)$. Except the OPAL experiment, there is no search for the final state with a tau lepton plus light quarks. Therefore, it seems that we cannot deny the possibility of a low-mass LQ based on previous searches without a global fit. On the other hand, we will show that low-energy flavour physics [66-69] can impose strong constraint on combinations of $\lambda_{l q}$ and $m_{\mathrm{LQ}}$ in section 2. We can only set upper limits on $\lambda_{l q}$ for a fixed LQ mass or vice versa. Given these difficulties, model-independent methods to search for LQs are highly necessary.

For the low mass LQs (less than $200 \mathrm{GeV}$ ), we find that the nuclear ultra-peripheral collision (UPC) is an ideal place to perform such searches. In UPCs, relativistic nuclei collide at impact parameters $(b)$ that are so large that there are no hadronic interactions. Thus it provides a unique opportunity to study photon-photon interactions. Refs. [71, 72] are two good reviews about the physics of UPCs. Given high photon flux at low energies (proportional to the 4-th power of the nucleus charge) and much cleaner collision environment, we propose to search for the LQ pair production in Pb-Pb UPC events. Most of previous searches use the property that LQs carry color charge. This proposal uses the property that LQs have electric charge, and is much less model-dependent compared to the existing searching strategies. Taking the scalar LQ with an electric charge $|q|=\frac{4}{3} e$, denoted as $S_{3}$ as in the review [32], as example, a feasibility study using all possible $l l q q(l=e, \mu, \tau$, $q=d, s, b$ and charge conjugation is always assumed) final states is conducted based on the performance of the ATLAS detector in Run 2 and Pb-Pb UPCs at the center-of-mass (c.m.) energy $\sqrt{s}=5.02 \mathrm{TeV}$.

In section 2, the constraints on the LQ mass and coupling constant from the experiments and the low-energy flavour physics are reviewed. In section 3, a much less modeldependent method is proposed to search for LQ pair production, which process dominates if the coupling strength of the LQ to the fermions is not big $(\lesssim 0.1$ as shown in figure 1$)$. 
It is worth emphasizing that the proposed method itself is also applicable to search for high-mass LQs in $p-p$ collisions as long as the pair production mechanism dominates. In section 4, we describe the cross sections in UPCs and the preparation of MC samples. In section 5, we elaborate the selection criteria and the analysis strategy. In section 6 , we will analyze the dominant systematic uncertainties and propose some solutions. In section 7 , we will describe the statistical interpretation scheme and present the sensitivity results. In section 8, we apply the method to the available $p$ - $p$ dataset from ATLAS [56] to investigate the possibility of excluding a $100 \mathrm{GeV}$ LQ unambiguously. Section 9 is a short summary.

\section{The bounds from the experimental searches and low-energy flavour physics}

\subsection{A robust lower mass bound from the OPAL experiments}

For a LQ, both its mass and coupling constant are unknown. The partial width of LQ $\rightarrow$ $l+q$ is

$$
\Gamma_{l q}=\frac{\lambda_{l q}^{2} m}{C \pi}
$$

where $C=16$ for a scalar LQ and 24 for a vector LQ. The LQ would decay outside a detector for a mass of $100 \mathrm{GeV}$ and a coupling constant of less than $10^{-8}$. Since a LQ carries both electric and color charge, a long-lived one would interact with the detector matter and may appear like a $b$-tagged jet. There is no dedicated search for such objects. If the lifetime is not too long, some direct searches are summarized in table 1. Among these results, two of the OPAL measurements [41, 42] seem to use the weakest assumption, which forbids a LQ to couple to different generations of leptons, but allow to couple to all possible quarks. In addition, it used the pair production in $e^{+} e^{-}$collisions. The pair production dominates if $\lambda \lesssim 10^{-2}$ and its cross section does not depend upon $\lambda$. Collecting the branching fractions, we can naively interpret their results in a different way (taking the LQ with the electric charge $|q|=\frac{4}{3} e$ and the measurement [42] as example).

$$
\begin{aligned}
& \sigma(m) B_{e x}^{2}<\sigma(100 \mathrm{GeV}), \\
& \sigma(m) B_{\mu x}^{2}<\sigma(101 \mathrm{GeV}), \\
& \sigma(m) B_{\tau x}^{2}<\sigma(99 \mathrm{GeV}),
\end{aligned}
$$

where $\sigma(m)$ is the production cross section of a pair of LQs with a mass $m$ in the process $e^{+} e^{-} \rightarrow \mathrm{LQ} \overline{\mathrm{LQ}} ; B_{l x}$ is the branching fraction of LQ $\rightarrow l x$ with $x=d, s, b$ for a fixed lepton $l$. Using $B_{e x}+B_{\mu x}+B_{\tau x}=1$ and the Cauchy-Schwarz inequality, we have

$$
\begin{aligned}
\sigma(m)\left(B_{e x}+B_{\mu x}+B_{\tau x}\right)^{2} & \leq \sigma(m) 3\left(B_{e x}^{2}+B_{\mu x}^{2}+B_{\tau x}^{2}\right) \\
& <3(\sigma(100 \mathrm{GeV})+\sigma(101 \mathrm{GeV})+\sigma(99 \mathrm{GeV})) \\
& <9 \sigma(99 \mathrm{GeV}) .
\end{aligned}
$$

Using the cross section formula in ref. [42] and the highest collision energy, a conservative bound is obtained.

$$
m>80 \mathrm{GeV} .
$$


This interpretation neither considers the shape information of the invariant mass reconstructed from a lepton candidate and a jet, nor considers the fact that there are multiple collision energy points with different luminosities. It does not take into account the contribution from LQ $\rightarrow \tau+q$ with a subsequent leptonical $\tau$ decay in the eeqq or $\mu \mu q q$ signal region, either. A full consideration shall give a better bound (but would not exceed $\sim 100 \mathrm{GeV}$ anyway). Thus we claim this is a robust bound with little model dependence.

A recent ATLAS measurement looked at the final states $e q$ and $\mu q$ with $q$ being all possible quarks except the top quark and searched for the LQ mass above $400 \mathrm{GeV}$ [57]. Exclusion regions as a function of the LQ mass and the branching fraction is reported (more details see [57]). Unfortunately, there seems no search for the final state with a tau lepton and light quarks except the OPAL experiment. It is difficult to exclude LQs with higher mass without assumptions.

Other experiments as summarized in table 1 either focused on only specific final state or made very strong assumptions. No further bounds can be obtained without a combined fit.

\subsection{The bounds from low-energy flavour physics}

Low-energy flavour physics like rare meson decays and lepton decays can provide stringent bounds on combinations of the coupling constant and mass [66-69]. In most cases, a LQ propagator would inevitably couple to a lepton and a quark on both ends. The extra amplitude induced by a LQ would be proportional to a factor of $\lambda_{l q} \lambda_{l^{\prime} q^{\prime}} / m_{\mathrm{LQ}}^{2}$ if the involved energy scale is much smaller than the LQ mass. This factor is invariant under the scaling transformation $\lambda \rightarrow k \lambda$ and $m_{\mathrm{LQ}} \rightarrow k m_{\mathrm{LQ}}$. Here we quote a few bounds from the literature. For $K_{L} \rightarrow e^{ \pm} \mu^{\mp}$, ref. [67] obtained

$$
|\lambda|^{2}<6 \times 10^{-7}\left(\frac{m_{\mathrm{LQ}}}{100 \mathrm{GeV}}\right)^{2} .
$$

For the $\mu^{-} \rightarrow e^{-}$conversion on the Titanium [70], ref. [68] obtained

$$
\left|\lambda_{\mu q} \lambda_{e q}\right|<1.7 \times 10^{-7}\left(\frac{m_{\mathrm{LQ}}}{100 \mathrm{GeV}}\right)^{2} .
$$

Interestingly, the neutral meson $\left(K^{0}, D^{0}, B^{0}\right)$ mixing is found to be able to constrain $\lambda^{2} / m_{\mathrm{LQ}}$. A recent calculation [69] gives roughly (the exact bound is model-dependent)

$$
|\lambda|^{2}<2.7 \times 10^{-3} \frac{m_{\mathrm{LQ}}}{100 \mathrm{GeV}} .
$$

Due to the scaling invariance above, one cannot provide definite bounds on a LQ's mass due to the mixing with the coupling constant. The existing anomalies in $B$-meson decays need significant contribution from new physics. The bounds based on those anomalies are highly model-dependent. We shall not expand the discussion here. In order to effectively bound a LQ's mass without touching its coupling to the fermions, we shall consider the physics processes where only LQs and gauge bosons are involved. For example, we can measure the differential cross section of $\gamma \gamma \rightarrow \gamma \gamma, \gamma \gamma \rightarrow g g$ or $g g \rightarrow \gamma \gamma$. Both the SM particles and LQs contribute at loop level. The first process is the cleanest, but the first 
and the second ones shall only be sensitive to low-mass LQs in heavy-ion collisions. The third one might be sensitive to high-mass LQs in $p-p$ collisions, though it suffers from the irreducible background $q \bar{q} \rightarrow \gamma \gamma$. The resonance effect near the threshold of a pair of LQs shall enhance the sensitivity. Anyway, the method presented in next section is more available and straightforward though more experimental efforts are needed.

\section{A model-independent searching method using the LQ pair production}

The LQ, $S_{3}$, considered in this feasibility study has a charge $|q|=\frac{4}{3} e$ and is allowed to decay to all possible $l q$ modes $(l=e, \mu, \tau$ and $q=d, s, b)$. The pair production in $p-p$ collisions and $\mathrm{Pb}-\mathrm{Pb}$ UPCs dominate if the coupling constant is not too big (e.g. $\lambda \lesssim 0.1$ as shown in figure 1). This is consistent with the bounds from the low-energy physics in last section. The cross section of pair production does not depend on the coupling constant, but on the branching fractions of the decay modes. Thus we consider all possible final states, namely, eejj, e $\mu j j, e \tau_{\text {had }} j j, \mu \mu j j, \mu \tau_{\text {had }} j j$, and $\tau_{\text {had }} \tau_{\text {had }} j j$, where $\tau_{\text {had }}$ denotes a hadronic tau decay candidate and $j$ denotes a jet candidate. We do not attempt to identify the jet flavour for simplicity. For each final state, all LQ's contributions are considered. For example, all $\mathrm{LQ} \rightarrow e q / \mu q / \tau q$ decays contribute in the $e \mu j j$ final state.

Three floating parameters, $\kappa, B_{e x}$ and $B_{\mu x}$, are introduced in the statistical interpretation. The branching fraction $B_{\tau x}$ is constrained to be $1-B_{e x}-B_{\mu x} . \kappa$ is the ratio of the measured cross section to the model prediction. We further impose the conditions $0 \leq B_{e x}, B_{\mu x} \leq 1$ and $B_{e x}+B_{\mu x} \leq 1$. For a given LQ mass, the measurement can determine the upper limit at $95 \%$ confidence level (C.L.) of $\kappa$. If the upper limit is less than 1 , then a LQ with the mass is excluded.

The method is model-independent in the sense that it allows a LQ to couple to all possible fermions.

\section{Cross sections and MC simulation}

\subsection{Cross sections in UPC}

For a nucleus $A$, the cross section for the process $A+A \rightarrow A+A+X$ is

$$
\sigma_{X}=\int d k_{1} d k_{2} \frac{d^{2} L_{\gamma \gamma}}{d k_{1} d k_{1}} \sigma^{\gamma \gamma \rightarrow X}\left(k_{1}, k_{2}\right),
$$

where $\sigma^{\gamma \gamma \rightarrow X}\left(k_{1}, k_{2}\right)$ is the production cross section of the final state $X$ in two-photon interactions with energy $k_{1}$ and $k_{2} ; d^{2} L_{\gamma \gamma} / d k_{1} d k_{2}$ is the corresponding two-photon luminosity.

$$
\frac{d^{2} L_{\gamma \gamma}}{d k_{1} d k_{2}}=\int_{b_{1}>R_{A}} \int_{b_{2}>R_{A}} d^{2} b_{1} d^{2} b_{2} \frac{d^{3} N_{\gamma}}{d k_{1} d^{2} b_{1}} \frac{d^{3} N_{\gamma}}{d k_{2} d^{2} b_{2}}
$$

where $R_{A}$ is the nucleus radius; and $d^{3} N_{\gamma} / d k d^{2} b$ is the photon flux from a nucleus at a distance $b$. For a charge $Z$ nucleus with velocity $\beta$ and Lorentz boost factor $\gamma$, the photon flux is

$$
\frac{d^{3} N_{\gamma}}{d k d^{2} b}=\frac{Z^{2} \alpha x^{2}}{\pi^{2} k b^{2}}\left[K_{1}^{2}(x)+\frac{K_{0}^{2}(x)}{\gamma}\right],
$$




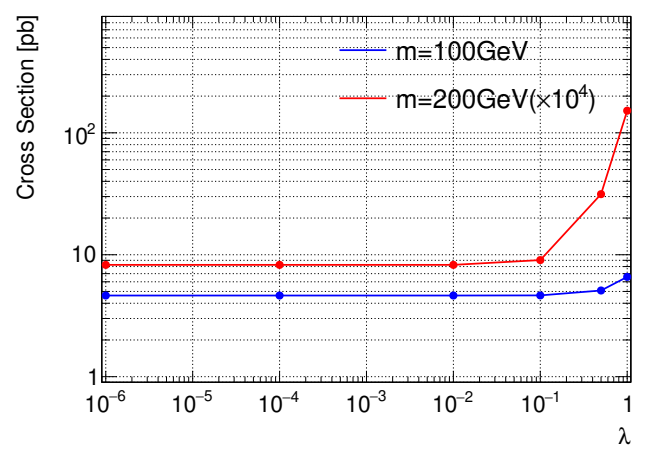

Figure 1. Fiducial cross section of $\mathrm{Pb}+\mathrm{Pb} \rightarrow \mathrm{Pb}+\mathrm{Pb}+e^{+} e^{-} d \bar{d}$ driven by the leptoquark $S_{3}$ in ultraperipheral collisions at $\sqrt{s}=5.03 \mathrm{TeV}$ as a function of the coupling constant $\lambda_{e d}$ with a mass of $100 \mathrm{GeV}$ (blue curve) or $200 \mathrm{GeV}$ (red curve, scaled by a factor of $10^{4}$ ).

where $x=\frac{k b}{\beta \gamma}$ and $K_{0}(x)$ and $K_{1}(x)$ are modified Bessel functions. The photon flux decreases exponentially above the cut off $k_{\max } \approx \frac{\gamma}{R_{A}}$.

In eq. (4.2), we have assumed that the probability of not having a hadronic interaction, denoted by $P_{0 \text { had }}(b)$, is 0 if $b<R_{A}$ and 1 if $b>R_{A}$. In general, we can replace the integration $\int_{b>R_{A}} \cdots d^{2} b$ in eq. (4.2) by $\int_{b=0}^{\infty} P_{0 \text { had }}(b) \cdots d^{2} b$. Using a more complicated description of $P_{0 \text { had }}(b)$ leads to a $10-15 \%$ difference in the flux [71].

\subsection{MC sample preparation}

The MadGraph 5 [73] is used to compute cross sections by convolving the WeizäckerWilliams equivalent photon flux (eq. (4.3)) of the nucleus with the elementary two-photon cross section, and to produce the signal and background samples. The parton showers are simulated with Pythia 8 [74] and the detector response is simulated with Delphes 3 [75] based on the Run 2 performance of the ATLAS detector.

The signal model is obtained from [76] and documented in ref. [77]. The LQ of interest in this work is the scalar boson $S_{3}$, which is allowed to couple to all charged leptons and down-type quarks. Six signal samples corresponding to the processes $\gamma \gamma \rightarrow S_{3} \bar{S}_{3} \rightarrow$ eeqq/e $\mu q q / e \tau q q / \mu \mu q q / \mu \tau q q / \tau \tau q q$ are produced with a mass $100 \mathrm{GeV}$ and all coupling constant fixed at $10^{-2}$. The possible interference effect with the SM process is not considered. The fiducial region at generator level is defined as $p_{\mathrm{T}}(l)>13 \mathrm{GeV}, p_{\mathrm{T}}(j)>15 \mathrm{GeV}$, $|\eta(l)|<2.5,|\eta(j)|<5, \Delta R(j, l)>0.3$ and $\Delta R(j, j)>0.3$, where $p_{\mathrm{T}}, \eta$ and $\Delta R$ represent the transverse momentum, rapidity and the angular distance, ${ }^{1}$ respectively.

Figure 1 shows the fiducial cross section of $\mathrm{Pb}+\mathrm{Pb} \rightarrow \mathrm{Pb}+\mathrm{Pb}+e^{+} e^{-} d \bar{d}$ as a function of the coupling constant $\lambda_{e d}$. Little dependence is seen for $\lambda_{e d} \lesssim 0.1$. The cross section used in the normalization does not consider the branching fractions, which will be treated as the parameters of interest in the statistical interpretation, and is scaled by a factor of $10^{-2}$ in the plots in section 5 just for better illustration.

\footnotetext{
${ }^{1}$ The angular distance between two objects is defined as $\Delta R(a, b) \equiv \sqrt{(\eta(a)-\eta(b))^{2}+(\phi(a)-\phi(b))^{2}}$, where $\eta$ and $\phi$ are the rapidity and azimuthal angle, respectively.
} 
The dominant background is from the SM process $\gamma \gamma \rightarrow l^{+} l^{-} q \bar{q}$. It is irreducible. The background events with multiple leptons or multiple jets are also considered and their contribution is minor. In addition, the background from $\mathrm{Pb}-\mathrm{Pb}$ hadronic interactions is inevitable. It is very difficult to simulate this background in reality. We usually design tight cuts to suppress it and estimate it in a data-driven way. We assume this background is negligible after the event selection in next section.

\section{Event selection and observable reconstruction}

Full reconstruction of all 4 objects is not desirable because of low efficiency. We require two lepton candidates and at least one jet candidates to be reconstructed. A charged lepton candidate $(e / \mu)$ is required to have transverse momentum $p_{\mathrm{T}}>15 \mathrm{GeV}$ and rapidity $|\eta|<2.5$. A hadronic tau candidate $\tau_{\text {had }}$ is required to have $p_{\mathrm{T}}>20 \mathrm{GeV}$ and $|\eta|<2.5$. The associated number of charged tracks is required to be 1 or 3 with unit sum of charges. A jet candidate denoted by $j$ is required to have $p_{\mathrm{T}}>20 \mathrm{GeV}$ and $|\eta|<5.0$.

An overlapping removal is performed according to the priority order $\mu \rightarrow e \rightarrow \tau_{\text {had }} \rightarrow j$. We first select muon candidate and then select electron candidate. The electron candidate is required not to overlap with the muon candidate within the angular distance $\Delta R=0.4$. $\tau_{\text {had }}$ candidate is required not to overlap with electron and muon candidates within the same angular distance. Similarly the $j$ candidate is required not to overlap with $\mu, e$ and $\tau_{\text {had }}$ candidates. 12 signal regions (SR) are designed below $(x=1,2)$.

- "eexj" signal region: 2 electron candidates with opposite charge sign and $x$ jets.

- "emuxj" signal region: 1 electron candidate and 1 muon candidate with opposite charge sign and $x$ jets.

- "etaxj" signal region: 1 electron candidate and $1 \tau_{\text {had }}$ candidates with opposite charge sign and $x$ jets.

- "mumuxj" signal region: 2 muon candidates with opposite charge sign and $x$ jets.

- "mutaxj" signal region: 1 muon candidate and $1 \tau_{\text {had }}$ candidate with opposite charge sign and $x$ jets.

- "tataxj" signal region: $2 \tau_{\text {had }}$ candidate and $x$ jets. For the signal region "tata $2 \mathrm{j}$ ", the missing transverse energy ${ }^{2} E_{\mathrm{T}}^{\mathrm{miss}}$ is required to be greater than $10 \mathrm{GeV}$ to suppress the multi-jet background.

If we have real data samples, we can apply further requirements to suppress the background due to the hadronic $\mathrm{Pb}-\mathrm{Pb}$ interactions: 1) no additional charged tracks in the inner detector and no additional energy deposit from neutral particles in the calorimeters as used in refs. [78, 79]; 2) no significant energy deposit in the Zero Degree Calorimeters [80] for the ATLAS detector (because the Pb nuclei are intact in UPCs) as used in ref. [81];

\footnotetext{
${ }^{2}$ The missing transverse energy is defined as negative vectorial sum of all visible objects' transverse momenta.
} 
3) adoption of multi-variate-analysis (MVA) methods $[85,86]$ to separate signal events from background events. In appendix $\mathrm{A}$, it is demonstrated that the signal purity in $\mathrm{Pb}-$ $\mathrm{Pb}$ collisions is much better than that in $p-p$ collisions.

The signal is identified as a peak structure in the invariant mass spectrum of a lepton and a jet candidates. For the events with only one jet, the jet is used twice to reconstruct the LQ mass. For the events with two jets, the best alignment is determined by minimizing their relative mass difference, namely,

$$
\min \frac{\left|m\left(l_{1} j_{1}\right)-m\left(l_{2} j_{2}\right)\right|}{m\left(l_{1} j_{1}\right)+m\left(l_{2} j_{2}\right)} .
$$

The subscript associated with the lepton candidate represents the charge sign, namely, $l_{1,2}=l^{ \pm}$, in the signal regions with the same flavour leptons (ee $\mathrm{j}$, mumuxj and tataxj). Otherwise the subscript represents the lepton flavour. For the etax $\mathrm{j}$ and mutax signal regions, we assume that the missing transverse energy is due to the neutrinos from the tau decays and thus added to the $\tau_{\text {had }}$ candidate, namely,

$$
\begin{aligned}
& p_{x}(\tau)=p_{x}\left(\tau_{\text {had }}\right)+E_{\mathrm{T}}^{\text {miss }} \cos \phi^{\text {miss }}, \\
& p_{y}(\tau)=p_{y}\left(\tau_{\text {had }}\right)+E_{\mathrm{T}}^{\text {miss }} \sin \phi^{\text {miss }}, \\
& p_{z}(\tau)=p_{z}\left(\tau_{\text {had }}\right)+E_{\mathrm{T}}^{\text {miss }} \sinh \eta\left(\tau_{\text {had }}\right),
\end{aligned}
$$

where $\phi^{\text {miss }}$ is the azimuthal angle of the missing transverse energy; and the collinear approximation, which assumes that the flight direction of the invisible neutrino(s) in a tau decay is the same as that of the visible tau decay products [82, 83], is used in eq. (5.4).

Assuming a luminosity of $1 \mathrm{fb}^{-1}$, figure $2-7$ show the distribution of the invariant mass of a lepton and a jet candidates in all signal regions. We can see that the $S_{3}$ resonance is well reconstructed, which also confirms that the resonant production of a $S_{3}$ pair dominates. Other features in these distributions are also expected: 1) the mass resolution of $m(e j)$ and $m(\mu j)$ is better than that of $m(\tau j) ; 2)$ The resolution of $m\left(\tau_{\text {had }} j\right)$ is even worse because it is only a partial reconstruction of the $S_{3}$ resonance; 3 ) the background level in the emu2j signal region is quite low because lepton flavour is conserved in the SM.

In order to improve the sensitivity to the mode $\mathrm{LQ} \rightarrow \tau q$, two mass reconstruction methods are tried in the tata2j signal region. One method is using the collinear approximation (technical details can be found in refs. [82, 83]), the other method is from ref. [83] which reconstructs the mass by sampling the neutrino(s)' momentum distribution in the center-of-mass frame of the tau lepton. The two mass variables, $m_{\text {coll }}$ and $m_{\text {xlg }}$, are shown in figure 8 . The latter mass $m_{\mathrm{xlg}}$ has a better resolution and accurately reconstructs the $S_{3}$ mass. Thus it is used as the final observable for the tata $2 \mathrm{j}$ signal region.

\section{$6 \quad$ Systematical uncertainties}

The systematical uncertainties can be categorized into experimental uncertainties and theoretical uncertainties. Here is a summary of experimental uncertainties based on the ATLAS detector in Run 2 and main theoretical uncertainties. 

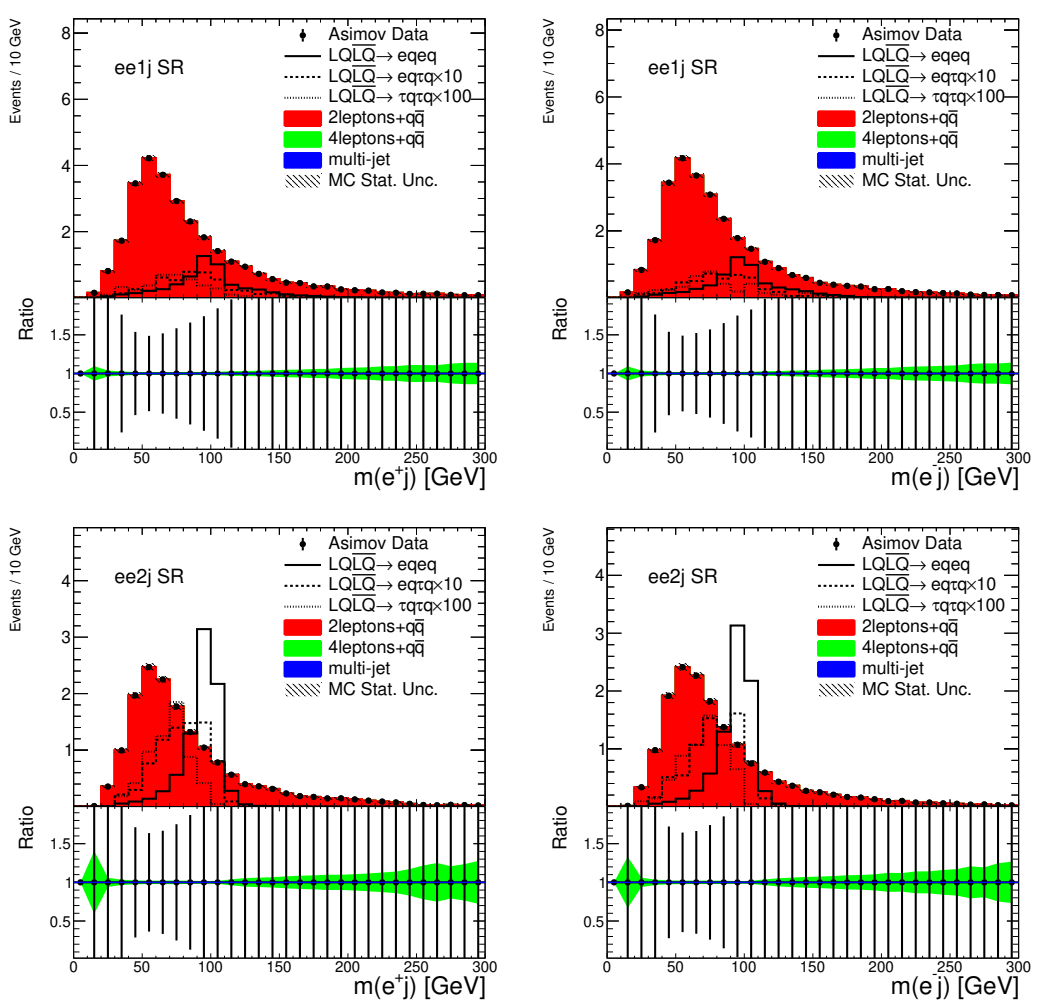

Figure 2. The distribution of the invariant mass of a lepton candidate and a jet candidate in the signal region "ee1j" (top) and "ee2j" (bottom). The left one is $m\left(e^{+} j\right)$ and the right one is $m\left(e^{-} j\right)$. The black points in the upper pads show the asimov data which is just the sum of all background events with uncertainties completely suppressed for better illustration. The ratio of the asimov data and the total background is shown in the lower pads, where the error bars on the black points represent the expected data uncertainty while the green bands represent the total MC statistical uncertainty.

- Luminosity uncertainty: $4 \%$, which is quoted from ref. [87] following the method in ref. [88].

- Object triggering, reconstruction and identification efficiencies: the performance of final object triggering, reconstruction and identification can found in ref. [89], ref. [90], ref. [91], ref. [92], and refs. [93, 94] for electron, muon, hadronic tau, missing transverse energy and jet, respectively. The efficiency uncertainties are usually expressed as a function of the transverse momentum. Table 2 shows the combined efficiency uncertainties (reconstruction and identification) for the transverse momentum range involved in this analysis. For jets, the dominant uncertainty is due to jet energy scale and energy resolution. In the transverse momentum range involved in this study, the systematical uncertainty is $5 \%$ on the energy scale and $6 \%$ on the energy resolution [94].

- Photon flux: there are a number of theoretic uncertainties in the photon flux calculations. Some of them need precise experimental measurements. One important uncertainty is about the probability of not having a hadronic interaction, $P_{0 \text { had }}(b)$. As 

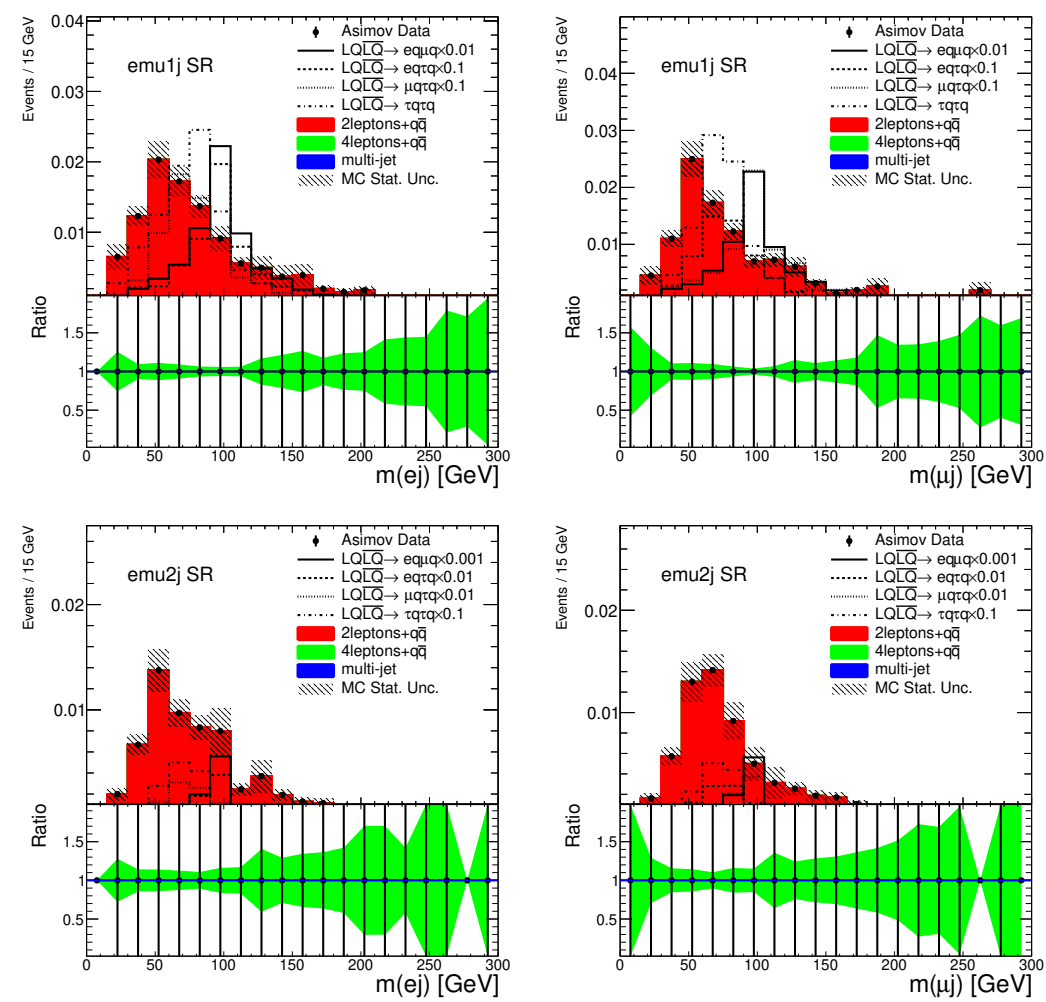

Figure 3. The distribution of the invariant mass of a lepton candidate and a jet candidate in the signal region "emu1j" (top) and "emu2j" (bottom). The left one is $m(e j)$ and the right one is $m(\mu j)$. The black points in the upper pads show the asimov data which is just the sum of all background events with uncertainties completely suppressed for better illustration. The ratio of the asimov data and the total background is shown in the lower pads, where the error bars on the black points represent the expected data uncertainty while the green bands represent the total MC statistical uncertainty.

\begin{tabular}{|ll|}
\hline Object & Combined efficiency uncertainty \\
\hline Electron & about $7 \%-3 \%$ for $p_{\mathrm{T}}=15-40 \mathrm{GeV}[89]$ \\
Muon & about $1 \%$ for $p_{\mathrm{T}}=15-40 \mathrm{GeV}[90]$ \\
Hadronic tau & about $5 \%$ for $p_{\mathrm{T}}=20-60 \mathrm{GeV}[91]$ \\
\hline
\end{tabular}

Table 2. The combined efficiency uncertainty (reconstruction and identification) of the final objects.

emphasized in the end of section 4.1, the different choices of $P_{0 \text { had }}(b)$ show a photon flux uncertainty of 10-15\% [71]. More discussions about these theoretic factors can be found in refs. [71, 72]. Photon flux uncertainty is directly related to the uncertainty of the signal production cross section. Here we present a data-driven estimation of this uncertainty. Because we have found a robust mass bound $m_{\mathrm{LQ}}>80 \mathrm{GeV}$ in section 2.1 and the irreducible background with $m(l j)<80 \mathrm{GeV}$ share the same final objects as the signal, thus the region with $m(l j)<80 \mathrm{GeV}$ can be used as a control region to constrain the photon flux uncertainty. 

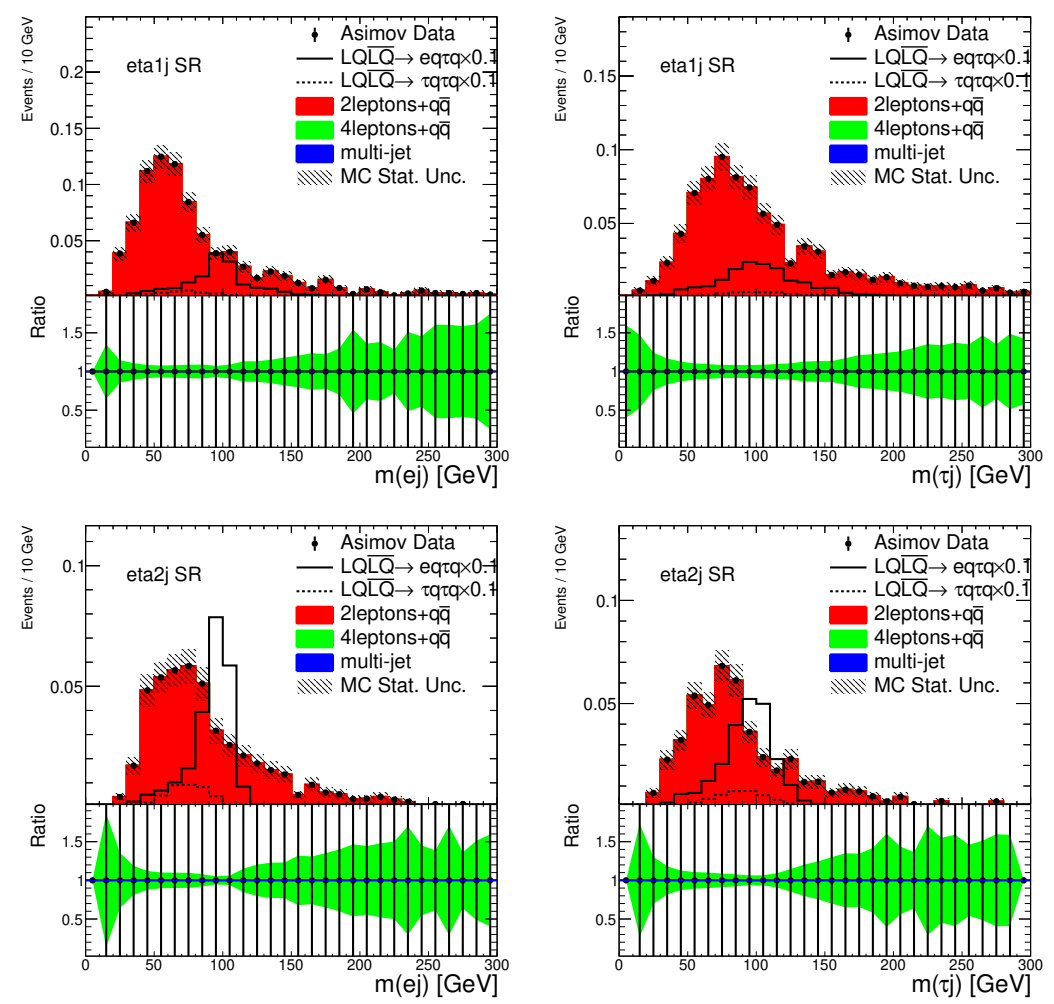

Figure 4. The distribution of the invariant mass of a lepton candidate and a jet candidate in the signal region "eta1j" (top) and "eta2j" (bottom). The left one is $m(e j)$ and the right one is $m(\tau j)$. The black points in the upper pads show the asimov data which is just the sum of all background events with uncertainties completely suppressed for better illustration. The ratio of the asimov data and the total background is shown in the lower pads, where the error bars on the black points represent the expected data uncertainty while the green bands represent the total MC statistical uncertainty.

- Uncertainty of the background due to $\mathrm{Pb}-\mathrm{Pb}$ hadronic interactions: the $\mathrm{MC}$ simulation of this background is unavailable and we have assumed it is negligible in the signal regions. One of the key features of photon-photon interactions in UPCs is that the net transverse momentum of the final state is very small. This feature can be used to estimate the $\mathrm{Pb}-\mathrm{Pb}$ hadronic background in the signal regions "ee $2 \mathrm{j}$ ", "emu2j", and "mumu2j" where no neutrinos are in the signal events. In other signal regions, we may use empirical functions to model it based on the fact that the background shape is expected to be smooth compared to the signal resonance structure.

- Jet flavour: no attempt is made to identify the jet flavour. The jet reconstruction efficiency may differ for different flavours. This can be estimated by MC and the systematical uncertainty can be controlled by studying the process of $\gamma \gamma \rightarrow q \bar{q}$. A more detailed analysis by categorizing the events into signal regions with light jets (from $u, d, s$ quarks), $c$-tagged jets and $b$-tagged jets will surely improve the sensitivity. But the methodology is the same. 

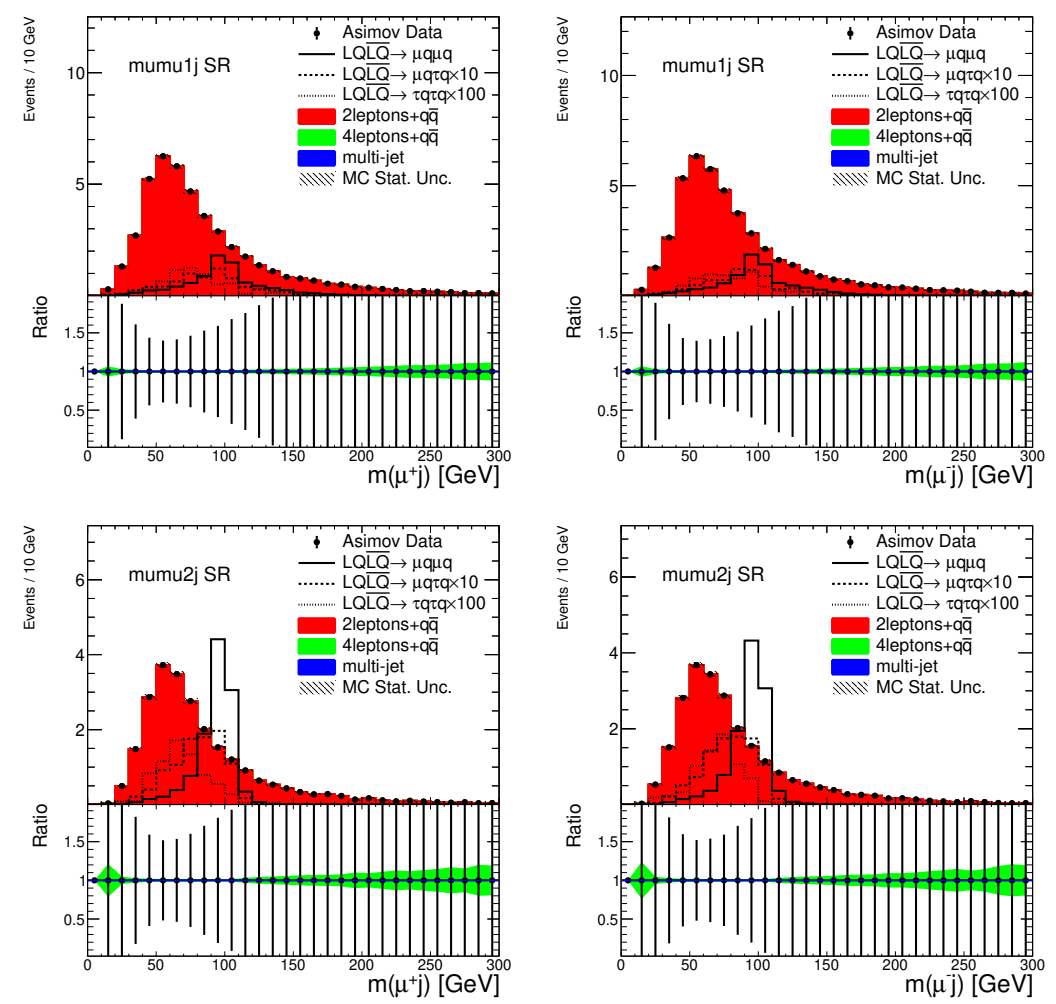

Figure 5. The distribution of the invariant mass of a lepton candidate and a jet candidate in the signal region "mumu1j" (top) and "mumu2j" (bottom). The left one is $m\left(\mu^{+} j\right)$ and the right one is $m\left(\mu^{-} j\right)$. The black points in the upper pads show the asimov data which is just the sum of all background events with uncertainties completely suppressed for better illustration. The ratio of the asimov data and the total background is shown in the lower pads, where the error bars on the black points represent the expected data uncertainty while the green bands represent the total MC statistical uncertainty.

\section{Statistical interpretation and sensitivity results}

A simultaneous binned likelihood fit is performed to the distributions shown in figures 2-8. The likelihood function is

$$
\mathcal{L}\left(\kappa, B_{e x}, B_{\mu x}\right)=\Pi_{i=1}^{12} \Pi_{j=1}^{N_{\text {bins }}^{i} P}\left(n_{j}^{i} \mid b_{j}^{i}+\kappa \sum_{k=1}^{N_{\text {sig }}^{i}} s_{j, k}^{i}\left(B_{e x}, B_{\mu x}\right)\right),
$$

where the index $i$ represents the $i$-th signal region; the index $j$ represent the $j$-th bin; the index $k$ represent the $k$-th signal mode; $N_{\text {bins }}^{i}$ represents the number of bin in the $i$-th signal region; $N_{\text {sig }}^{i}$ represent the number of signal modes in the $i$-th signal region; and $s_{j, k}^{i}\left(B_{e x}, B_{\mu x}\right)$ represents the yield contribution of the $k$-th signal mode to the $j$-th bin in the $i$-th signal region. The yield of each signal mode should be associated to the branching fractions appropriately. For example, we need to multiply the signal yield of $\mathrm{LQ} \overline{\mathrm{LQ}} \rightarrow e q \tau q$ by a factor of $2 B_{e x}\left(1-B_{e x}-B_{\mu x}\right)$. Furthermore, we should impose the constraint $0 \leq B_{e x}+B_{\mu x} \leq 1$ as stated in section 3 . 

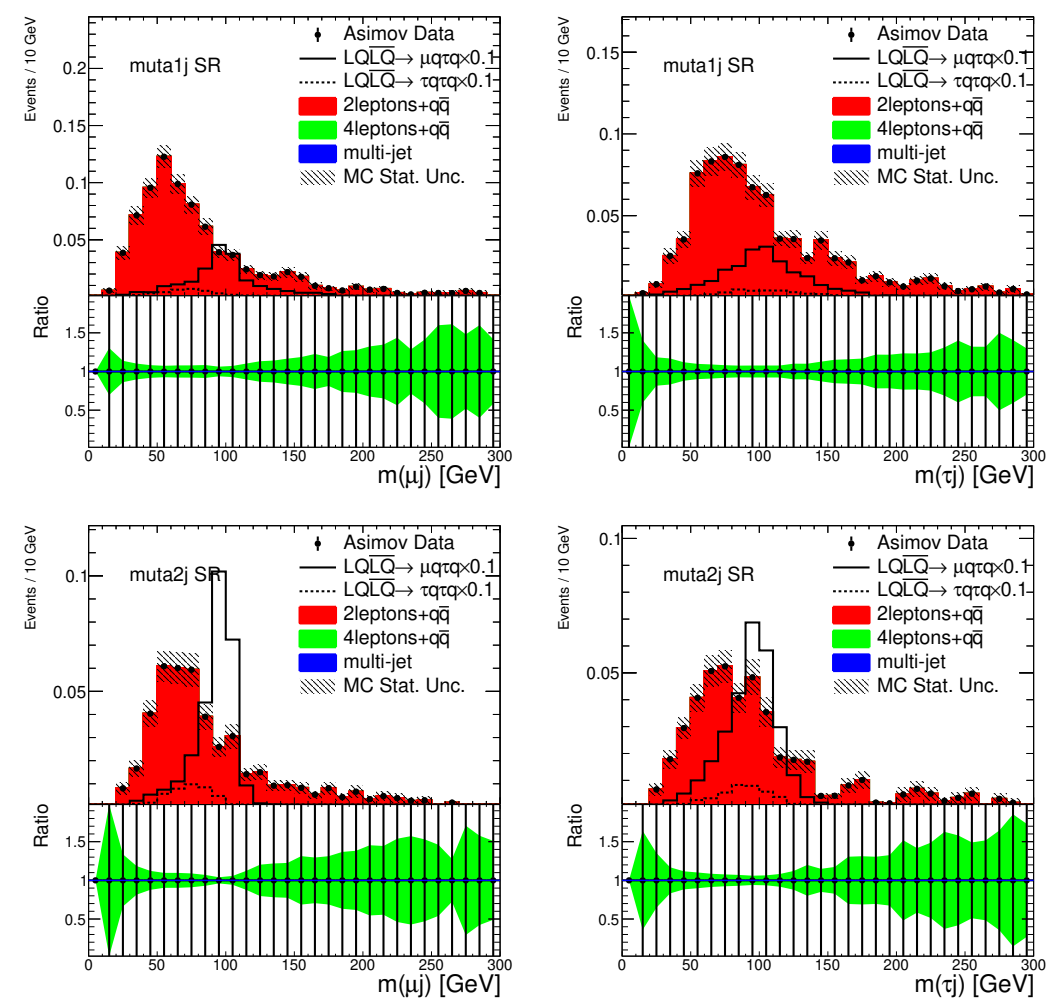

Figure 6. The distribution of the invariant mass of a lepton candidate and a jet candidate in the signal region "muta1j" (top) and "muta2j" (bottom). The left one is $m(\mu j)$ and the right one is $m(\tau j)$. The black points in the upper pads show the asimov data which is just the sum of all background events with uncertainties completely suppressed for better illustration. The ratio of the asimov data and the total background is shown in the lower pads, where the error bars on the black points represent the expected data uncertainty while the green bands represent the total MC statistical uncertainty.

\begin{tabular}{|lll|}
\hline$S_{3}$ mass & Upper limit of $\kappa$ (95\%C.L.) for $1 \mathrm{fb}^{-1}$ & Luminosity to exclude $\kappa=1$ \\
\hline $100 \mathrm{GeV}$ & $1.1 \times 10^{-2}$ & $4.0 \mathrm{pb}^{-1}$ \\
\hline
\end{tabular}

Table 3. Expected sensitivity to search for $S_{3}$ with a mass of $100 \mathrm{GeV}$ using $1 \mathrm{fb}^{-1}$ of Pb-Pb UPCs at $\sqrt{s}=5.02 \mathrm{TeV}$.

Allowing $B_{e x}$ and $B_{\mu x}$ to float, the left plot of figure 9 shows the $-2 \Delta \ln \mathcal{L}(\kappa) \equiv$ $-2 \ln \frac{\mathcal{L}(\kappa)}{\mathcal{L}_{\max }}$ as a function of $\kappa$ assuming the absence of new physics. We can see that $\kappa>1.1 \times 10^{-2}$ can be excluded at $95 \%$ confidence level for a dataset with the luminosity $L=1 \mathrm{fb}^{-1}$. To exclude $\kappa>1$, a dataset of $L \approx 4.0 \mathrm{pb}^{-1}$ is needed as shown in right plot of figure 9 . The sensitivity results are summarized in table 3 .

\section{Can we exclude a $100 \mathrm{GeV}$ LQ unambiguously?}

In this section, we allow a LQ to decay to all possible fermions and investigate the possibility of a $100 \mathrm{GeV}$ LQ with an electric charge $\frac{4}{3} e$ considering all available experimental constraints. The results from the H1 experiment [43-45], CDF experiment [46-48], and 

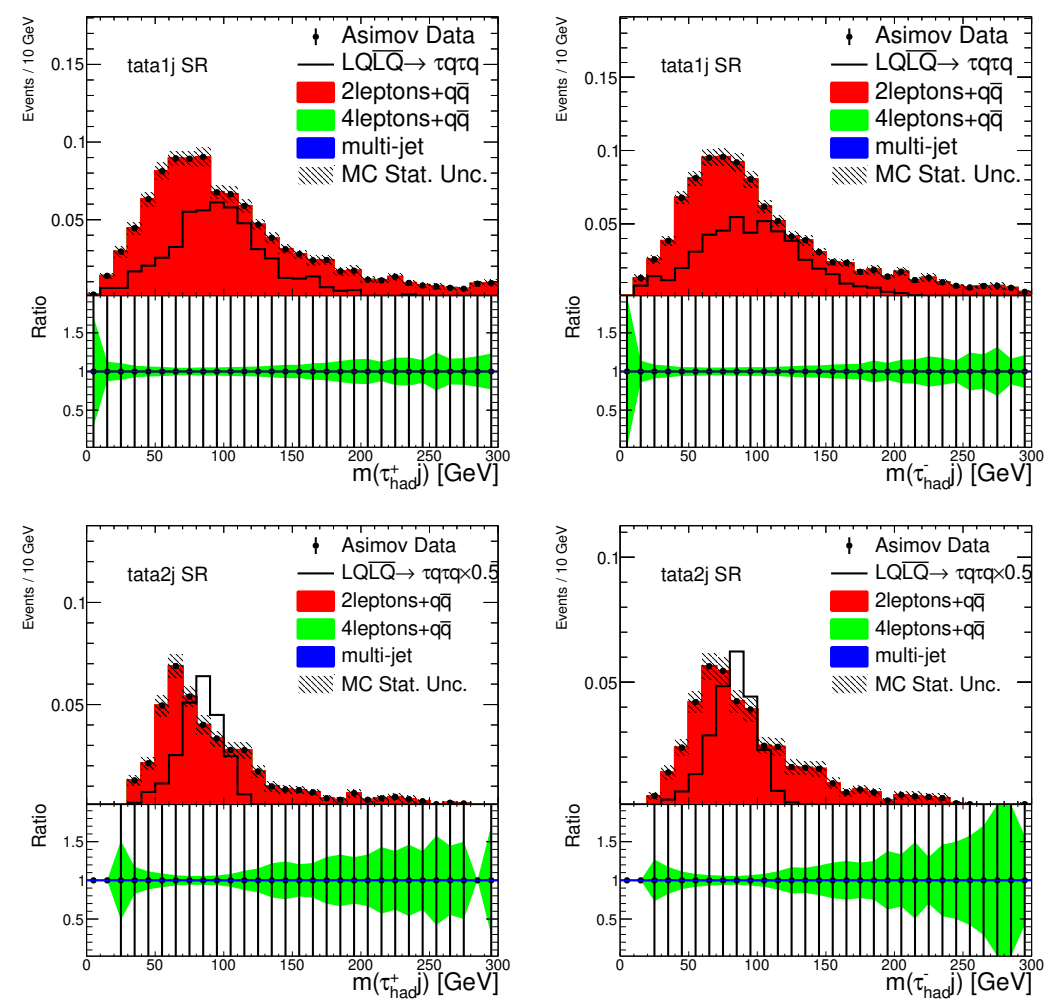

Figure 7. The distribution of the invariant mass of a lepton candidate and a jet candidate in the signal region "tata1j" (top) and "tata2j" (bottom). The left one is $m\left(\tau_{\text {had }}^{+} j\right)$ and the right one is $m\left(\tau_{\text {had }}^{-} j\right)$. The black points in the upper pads show the asimov data which is just the sum of all background events with uncertainties completely suppressed for better illustration. The ratio of the asimov data and the total background is shown in the lower pads, where the error bars on the black points represent the expected data uncertainty while the green bands represent the total MC statistical uncertainty.

ATLAS experiment [56] are used and summarized in table 4. We do not include other experiments which did not look at this mass region although they may potentially provide stronger constraints. Table I summarizes the available constraints with the orignal interpretation with the corresponding assumption. We need to reinterpret them to remove the assumption. If an experiment does not explicitly identify the flavour of a jet, we will always include either all light down-type quarks (denoted by $q=d, s$ ) or all down-type quarks (denoted by $Q=d, s, b)$ in the reinterpretation.

In the $\mathrm{H} 1$ experiment, a LQ is produced via the single production mode $e+p \rightarrow \mathrm{LQ}+X$. For LQ $\rightarrow l q$, the final yield is proportional to $\lambda_{e d}^{2} B_{l q}$ (we ignore the possible interference with the SM process). The constraints are then re-expressed as

$$
\begin{aligned}
& \lambda_{e d}^{2} B_{e q} \lesssim(0.01)^{2} \\
& \lambda_{e d}^{2} B_{\mu q}<2 \times(0.001)^{2} \\
& \lambda_{e d}^{2} B_{e q} \lesssim(0.006)^{2},
\end{aligned}
$$

which are from refs. [43-45], respectively. 

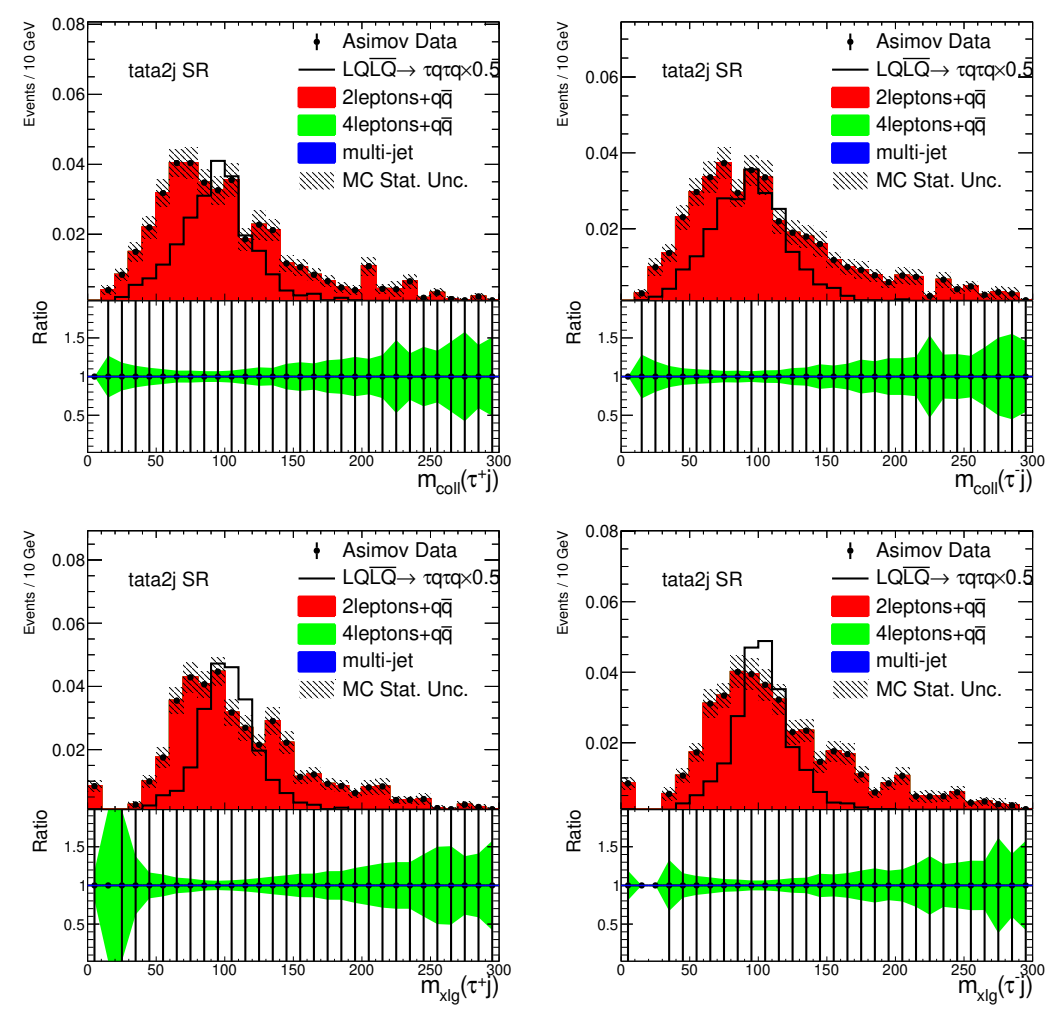

Figure 8. The distribution of the reconstructed mass of a lepton candidate and a jet candidate in the signal region "tata2j" based on the collinear approximation method (top) and the method in ref. [83] (Bottom). The left one is $m\left(\tau^{+} j\right)$ and the right one is $m\left(\tau^{-} j\right)$. The black points in the upper pads show the asimov data which is just the sum of all background events with uncertainties completely suppressed for better illustration. The ratio of the asimov data and the total background is shown in the lower pads, where the error bars on the black points represent the expected data uncertainty while the green bands represent the total MC statistical uncertainty.
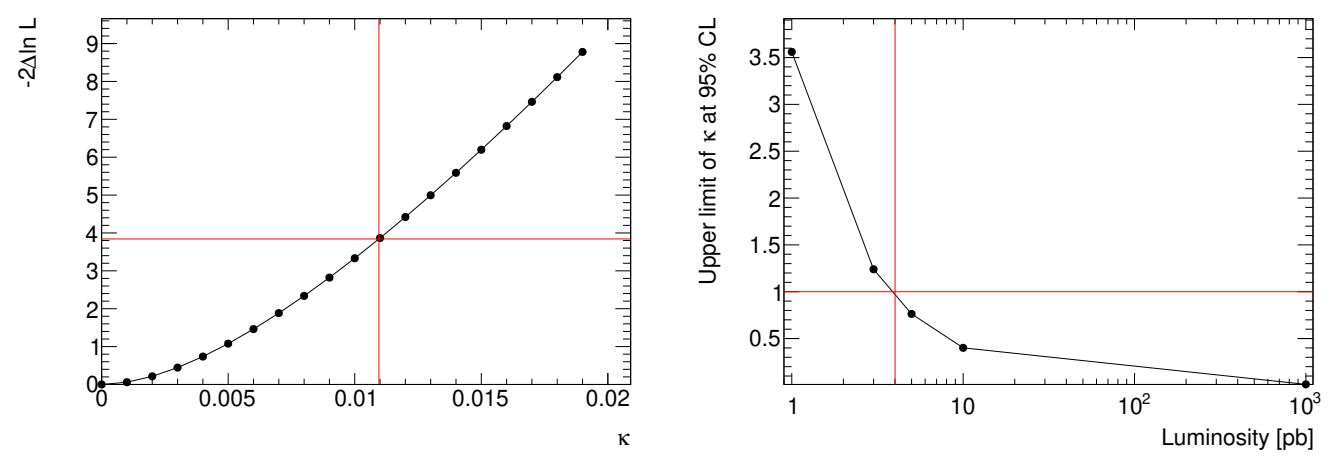

Figure 9. Left: the $-2 \Delta \ln \mathcal{L}$ as a function of $\kappa$ for a luminosity of $1 \mathrm{fb}^{-1}$. The red lines indicate the interval at $95 \%$ confidence level. Right: the upper limit of $\kappa$ at $95 \%$ confidence level as a function of the luminosity. The red lines indicate the luminosity corresponding to the upper limit of $\kappa$ being 1 . 


\begin{tabular}{|llll|}
\hline Exp. & Source & Original constraint (assumption) & Re-interpretation \\
\hline H1 [43] & Figure 13 in [43] & $\lambda_{e d} \lesssim 0.01\left(B_{e d}=1\right)$ & $\lambda_{e d}^{2} B_{e q}<0.01^{2}$ \\
H1 [44] & Figure 3 in [44] & $\lambda_{e d / \mu d} \lesssim 0.001\left(B_{e d / \mu d}=\frac{1}{2}\right)$ & $\lambda_{e d}^{2} \frac{B_{\mu q}}{\frac{1}{2}}<(0.001)^{2}$ \\
H1 [45] & Figure 3 in [45] & $\lambda_{e d} \lesssim 0.006\left(B_{e d}=1\right)$ & $\lambda_{e d}^{2} B_{e q} \lesssim(0.006)^{2}$ \\
CDF [46] & Table I in [46] & $\sigma<10 \mathrm{pb}\left(B_{\tau Q}=1\right)$ & $\sigma_{\mathrm{th}} B_{\tau Q}^{2}<10 \mathrm{pb}, \sigma_{\mathrm{th}}=9.8 \mathrm{pb}$ \\
CDF [47] & Table I in [47] & $\sigma<1.1 \mathrm{pb}\left(B_{e q}=1\right)$ & $\sigma_{\mathrm{th}} B_{e q}^{2}<1.1 \mathrm{pb}, \sigma_{\mathrm{th}}=11.13 \mathrm{pb}$ \\
CDF [48] & Table II in [48] & $\sigma<1.35 \mathrm{pb}\left(B_{\mu q}=1\right)$ & $\sigma_{\mathrm{th}} B_{\mu q}^{2}<1.35 \mathrm{pb}, \sigma_{\mathrm{th}}=11.13 \mathrm{pb}$ \\
ATLAS [56] & Figure 4 in [56] & Exclude 200-800 GeV (SG) & see text \\
\hline
\end{tabular}

Table 4. Reinterpretation of the experimental constraints on a $100 \mathrm{GeV}$ LQ with an electric charge $\frac{4}{3} e$. In the table, $q$ denotes the light down-type quarks $d$ and $s ; Q$ denotes all down-type quarks $d, s$ and $b$.

In the CDF experiment, a pair of LQs is produced in the reaction of $p \bar{p} \rightarrow \mathrm{LQ} \overline{\mathrm{LQ}}+X$. The final yield is proportional to the product of the pair-production cross section and the branching fractions. Using the cross section from the theory, we can obtain the constraints on the branching fractions, namely,

$$
\begin{aligned}
& B_{\tau Q}<1.01 \\
& B_{e q}<0.31 \\
& B_{\mu q}<0.35
\end{aligned}
$$

which are from refs. [46-48], respectively.

Based on the constraints above, it is impossible to exclude a $100 \mathrm{GeV} \mathrm{LQ}$ definitely because all couplings are not covered. The ATLAS and CMS experiments have also performed comprehensive searches for various LQs via either single- or pair-production mechanism. However, we find only ref. [56] from ATLAS has a public dataset which can be used to probe the existence of a $100 \mathrm{GeV} \mathrm{LQ}$ with an electric charge $\frac{4}{3} e$. The original motivation of ref. [56] is to search for a LQ decaying to $t \tau / b \nu$ or $b \tau / t \nu$. We will use their data with the final state $\tau_{\text {lep }} \tau_{\text {had }}$ plus one or more $b$-tagged jets, namely, figure 4 (middle left) from ref. [56] and figure $6 \mathrm{~b}$, figure $6 \mathrm{c}$ and figure $7 \mathrm{~b}$ in its auxiliary material [95]. The data with the $\tau_{\text {had }} \tau_{\text {had }}$ final state is not used due to the complicated trigger requirements which depend upon the real-time data-taking status.

Figure 10 shows the distribution of the pair mass of a tau-lepton candidate and its matched jet candidate in the signal region with one $b$-tagged jets (denoted by $\tau_{\text {lep }} \tau_{\text {had }} 1 j_{b}$ ) and signal region with two $b$-tagged jets (denoted by $\tau_{\text {lep }} \tau_{\text {had }} 2 j_{b}$ ). The matching criterion is to minimize the mass difference of the LQ pair candidate (see ref. [56]).

We allow all possible decay modes, and do not differentiate $e$ and $\mu$ or $d$ and $s$. We thus have four decay modes, LQ $\rightarrow b \tau, b l, q \tau, q l$ where $l=e, \mu$ and $q=d, s$ and 10 individual signal topologies from a LQ pair. They are also shown in figure 10. In preparing signal samples, we have tried our best to apply the same selection criteria as described in ref. [56]. Given that no significant deviation from the SM prediction is seen and following the methodology presented in this work, the upper limit at 95\% C.L. of the pair-production 

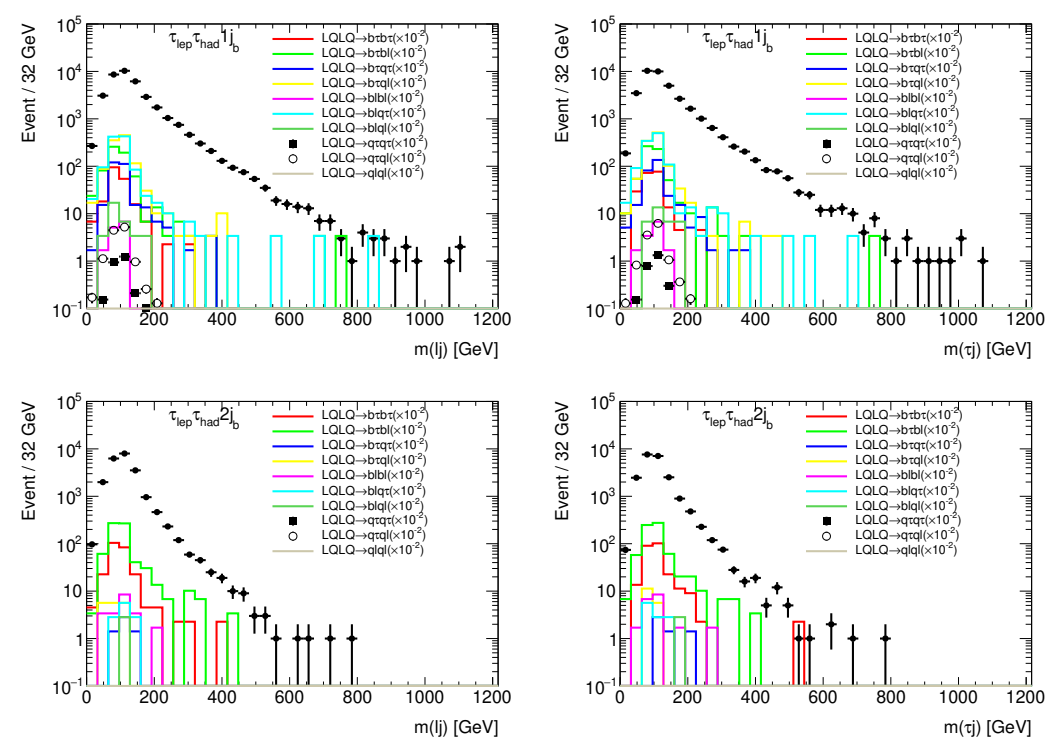

Figure 10. The distribution of the pair mass of a tau-lepton candidate and its mass-matched jet candidate in the signal region with one $b$-tagged jet (top) and two $b$-tagged jets (bottom). The left one is $m(l j)$ and the right one is $m(\tau j)$. The black points represent the data from refs. [56,95]. The open circles, black squares and histograms represent different signal topologies for a $100 \mathrm{GeV} \mathrm{LQ}$.

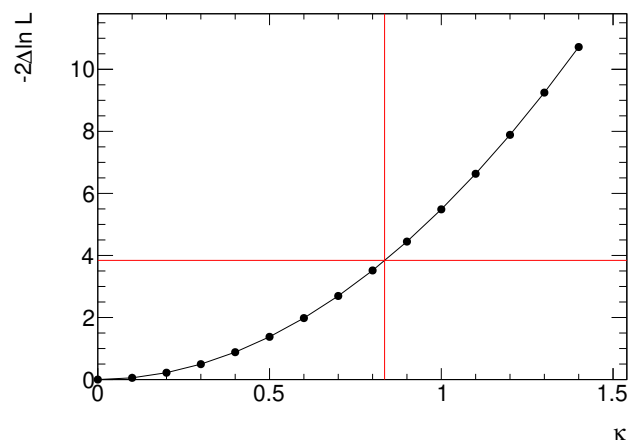

Figure 11. The $-2 \Delta \ln \mathcal{L}$ as a function of $\kappa$ for a $100 \mathrm{GeV} \mathrm{LQ}$. The red lines indicate the interval at $95 \%$ confidence level.

cross section modifier $\kappa$ is determined to be 0.83 as shown in figure 11, for which the CDF constraints in eq. (8.5)-(8.6) have been considered. It means that a LQ with a mass $100 \mathrm{GeV}$ is excluded at $95 \%$ C.L. under the general condition that a LQ can decay to all possible fermions.

It is worthwhile to make two comments: 1) the sensitivity is dominated by the modes $q \tau q \tau$ and $q \tau q l$ (marked as squares and open circles in figure 10) for which we should design a signal region without any $b$-tagged jet in reality; 2) ref. [56] claims to exclude a LQ up to $1030 \mathrm{GeV}$ assuming $B_{b \tau}=1$. It is likely that the exclusion region would shrink much dropping the assumption. Given the observation in this section, this work is better seen as a proof of concept in order to search for LQs in a least model-dependent way. 


\section{Summary}

The possibility of low-mass leptoquarks cannot be excluded due to the assumptions made in previous experimental measurements. In this work, we propose a least model-dependent method (assuming $\lambda \lesssim 0.1$ ) to search for them in the Pb-Pb UPCs thanks to the high photon flux and potentially low background contamination. It allows a LQ to couple to all possible lepton-quark pairs. Taking the scalar LQ $S_{3}$ as example, the feasibility is investigated using all $l l q q$ final states based on the Pb-Pb UPCs at $\sqrt{s}=5.02 \mathrm{TeV}$ and the performance of the ATLAS detector in Run 2. The LQ with a mass of $100 \mathrm{GeV}$ can be excluded at $95 \%$ confidence level using a dataset of $4.0 \mathrm{pb}^{-1}$. This proposal uses the property that LQs carry electric charge and is complementary to the searches in $p-p$ collisions. The method also works for searching for high-mass LQs in $p-p$ collisions as long as the pair production mechanism dominates.

\section{Acknowledgments}

I am greatly indebted to David d'Enterria for support with MadGraph and the theoretical discussions about UPC. I also wish to express my appreciation to Prof. Shan Jin for helping to resume my research career. Especially I would like to thank Fang Dai for her encouragement and financial support.

\section{A Signal purity comparison between in $\mathrm{Pb}-\mathrm{Pb}$ collisions and in $p$ - $p$ col- lisions}

In this appendix, we compare the signal purity between in $\mathrm{Pb}-\mathrm{Pb}$ collisions at $5.02 \mathrm{TeV}$ and in $p$ - $p$ collisions at $13 \mathrm{TeV}$ using the $b b \tau \tau$ final state (an experimental search see ref. [34]). The final objects are reconstructed in the same way as described in section 5 . The $b$-jet is reconstructed at a working point with an efficiency of $80 \%$. The events with one charged lepton candidate $(e$ or $\mu$ ), one hadronic tau decay candidate and one $b$-jet candidate are selected. The luminosity is $1 \mathrm{fb}^{-1}$ for $\mathrm{Pb}-\mathrm{Pb}$ collisions and $36 \mathrm{fb}^{-1}$ for $p-p$ collisions.

Figure 12 shows the distribution of the invariant mass of a hadronic tau candidate and a $b$-jet candidate. Both the production cross section in $\mathrm{Pb}-\mathrm{Pb}$ collisions and in $p-p$ collisions are scaled by a factor $10^{-2}$ for better illustration (not including the scaling factor as shown in the legend in figure 12). We can see that the signal-to-background ratio in $\mathrm{Pb}-\mathrm{Pb}$ collisions is around 50 times higher than that in $p-p$ collisions. The reason is that the $t \bar{t}$ background dominates in $p-p$ collisions while it is negligible in $\mathrm{Pb}-\mathrm{Pb}$ collisions (the cross section is about $1 \%$ that of $b b \tau \tau$ production in the $\mathrm{SM}$ ). This is one of the advantages of searching for low-mass LQs in Pb-Pb UPCs. 

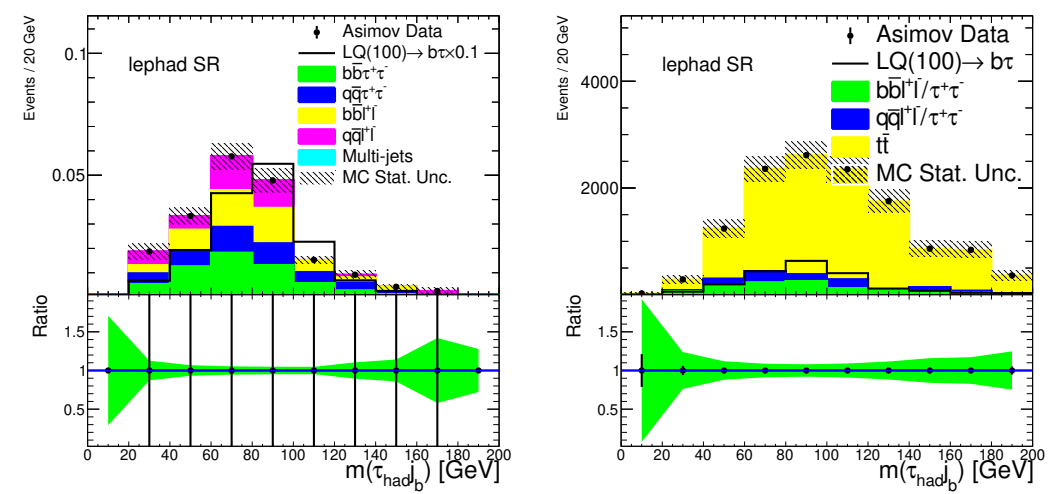

Figure 12. The distribution of the invariant mass of a hadronic tau candidate and a $b$-jet candidate in the $\mathrm{Pb}-\mathrm{Pb}$ collisions $(\mathrm{L})$ and the $p-p$ collisions $(\mathrm{R})$. The signal histograms are scaled by a factor of $10^{-2}$ besides the scaling factor in the legends. The black points in the upper pads show the asimov data which is just the sum of all background events with uncertainties completely suppressed for better illustration. The ratio of the asimov data and the total background is shown in the lower pads, where the error bars on the black points represent the expected data uncertainty while the green bands represent the total MC statistical uncertainty.

Open Access. This article is distributed under the terms of the Creative Commons Attribution License (CC-BY 4.0), which permits any use, distribution and reproduction in any medium, provided the original author(s) and source are credited.

\section{References}

[1] J.C. Pati and A. Salam, Unified Lepton-Hadron Symmetry and a Gauge Theory of the Basic Interactions, Phys. Rev. D 8 (1973) 1240 [INSPIRE].

[2] J.C. Pati and A. Salam, Lepton Number as the Fourth Color, Phys. Rev. D 10 (1974) 275 [Erratum ibid. 11 (1975) 703] [INSPIRE].

[3] H. Georgi and S.L. Glashow, Unity of All Elementary Particle Forces, Phys. Rev. Lett. 32 (1974) 438 [INSPIRE].

[4] H. Fritzsch and P. Minkowski, Unified Interactions of Leptons and Hadrons, Annals Phys. 93 (1975) 193 [INSPIRE].

[5] W. Buchmüller and D. Wyler, Constraints on SU(5) Type Leptoquarks, Phys. Lett. B 177 (1986) 377 [INSPIRE].

[6] S. Dimopoulos and L. Susskind, Mass Without Scalars, Nucl. Phys. B 155 (1979) 237 [INSPIRE].

[7] S. Dimopoulos, Technicolored Signatures, Nucl. Phys. B 168 (1980) 69 [InSPIRE].

[8] E. Farhi and L. Susskind, Technicolor, Phys. Rept. 74 (1981) 277 [INSPIRE].

[9] K.D. Lane and M.V. Ramana, Walking technicolor signatures at hadron colliders, Phys. Rev. D 44 (1991) 2678 [INSPIRE].

[10] B. Schrempp and F. Schrempp, Light Leptoquarks, Phys. Lett. B 153 (1985) 101 [InSPIRE]. 
[11] B. Gripaios, Composite Leptoquarks at the LHC, JHEP 02 (2010) 045 [arXiv:0910.1789] [INSPIRE].

[12] B. Gripaios, M. Nardecchia and S.A. Renner, Composite leptoquarks and anomalies in $B$-meson decays, JHEP 05 (2015) 006 [arXiv:1412.1791] [INSPIRE].

[13] E. Eichten and K.D. Lane, Dynamical Breaking of Weak Interaction Symmetries, Phys. Lett. $B 90$ (1980) 125 [INSPIRE].

[14] V.D. Angelopoulos, J.R. Ellis, H. Kowalski, D.V. Nanopoulos, N.D. Tracas and F. Zwirner, Search for New Quarks Suggested by the Superstring, Nucl. Phys. B 292 (1987) 59 [InSPIRE].

[15] G. Hiller and M. Schmaltz, $R_{K}$ and future $b \rightarrow$ sll physics beyond the standard model opportunities, Phys. Rev. D 90 (2014) 054014 [arXiv:1408.1627] [INSPIRE].

[16] M. Freytsis, Z. Ligeti and J.T. Ruderman, Flavor models for $\bar{B} \rightarrow D^{(*)} \tau \bar{\nu}$, Phys. Rev. $D 92$ (2015) 054018 [arXiv: 1506. 08896] [INSPIRE].

[17] M. Bauer and M. Neubert, Minimal Leptoquark Explanation for the $R_{D^{(*)}}, R_{K}$, and $(g-2)_{\mu}$ Anomalies, Phys. Rev. Lett. 116 (2016) 141802 [arXiv:1511.01900] [INSPIRE].

[18] L. Di Luzio and M. Nardecchia, What is the scale of new physics behind the B-flavour anomalies?, Eur. Phys. J. C 77 (2017) 536 [arXiv:1706.01868] [INSPIRE].

[19] D. Buttazzo, A. Greljo, G. Isidori and D. Marzocca, B-physics anomalies: a guide to combined explanations, JHEP 11 (2017) 044 [arXiv: 1706.07808] [INSPIRE].

[20] J.M. Cline, $B$ decay anomalies and dark matter from vectorlike confinement, Phys. Rev. D 97 (2018) 015013 [arXiv: 1710.02140] [INSPIRE].

[21] W. Buchmüller, R. Ruckl and D. Wyler, Leptoquarks in Lepton-Quark Collisions, Phys. Lett. B 191 (1987) 442 [Erratum ibid. 448 (1999) 320] [INSPIRE].

[22] A. Angelescu, D. Bečirević, D.A. Faroughy and O. Sumensari, Closing the window on single leptoquark solutions to the B-physics anomalies, JHEP 10 (2018) 183 [arXiv:1808.08179] [INSPIRE].

[23] D. Bečirević, I. Doršner, S. Fajfer, N. Košnik, D.A. Faroughy and O. Sumensari, Scalar leptoquarks from grand unified theories to accommodate the B-physics anomalies, Phys. Rev. D 98 (2018) 055003 [arXiv: 1806.05689] [INSPIRE].

[24] LHCb collaboration, Test of lepton universality in beauty-quark decays, arXiv:2103.11769 [INSPIRE].

[25] L.-S. Geng, B. Grinstein, S. Jäger, S.-Y. Li, J. Martin Camalich and R.-X. Shi, Implications of new evidence for lepton-universality violation in $b \rightarrow s \ell^{+} \ell^{-}$decays, arXiv:2103.12738 [INSPIRE].

[26] M. Du, J. Liang, Z. Liu and V.Q. Tran, A vector leptoquark interpretation of the muon $g-2$ and $B$ anomalies, arXiv:2104.05685 [INSPIRE].

[27] J. Kriewald, C. Hati, J. Orloff and A.M. Teixeira, Leptoquarks facing flavour tests and $b \rightarrow$ sll after Moriond 2021, in proceedings of the 55th Rencontres de Moriond on Electroweak Interactions and Unified Theories, 21-27 March 2021, online conference, Italy, arXiv: 2104.00015 [INSPIRE].

[28] H.M. Lee, Leptoquark option for B-meson anomalies and leptonic signatures, Phys. Rev. D 104 (2021) 015007 [arXiv:2104.02982] [INSPIRE]. 
[29] G. Hiller, D. Loose and I. Nišandžić, Flavorful leptoquarks at the LHC and beyond: spin 1, JHEP 06 (2021) 080 [arXiv:2103.12724] [INSPIRE].

[30] A. Angelescu, D. Bečirević, D.A. Faroughy, F. Jaffredo and O. Sumensari, On the single leptoquark solutions to the B-physics anomalies, arXiv:2103.12504 [INSPIRE].

[31] C. Cornella, D.A. Faroughy, J. Fuentes-Martín, G. Isidori and M. Neubert, Reading the footprints of the B-meson flavor anomalies, arXiv:2103.16558 [INSPIRE].

[32] I. Doršner, S. Fajfer, A. Greljo, J.F. Kamenik and N. Košnik, Physics of leptoquarks in precision experiments and at particle colliders, Phys. Rept. 641 (2016) 1 [arXiv: 1603.04993] [INSPIRE].

[33] CMS collaboration, Search for pair production of third-generation scalar leptoquarks and top squarks in proton-proton collisions at $\sqrt{s}=8 \mathrm{TeV}$, Phys. Lett. B 739 (2014) 229 [arXiv: 1408.0806] [INSPIRE].

[34] ATLAS collaboration, Searches for third-generation scalar leptoquarks in $\sqrt{s}=13$ TeV pp collisions with the ATLAS detector, JHEP 06 (2019) 144 [arXiv: 1902.08103] [INSPIRE].

[35] CMS collaboration, Search for singly and pair-produced leptoquarks coupling to third-generation fermions in proton-proton collisions at $\sqrt{s}=13 \mathrm{TeV}$, Phys. Lett. $B \mathbf{8 1 9}$ (2021) 136446 [arXiv:2012.04178] [INSPIRE].

[36] AMY collaboration, A search for leptoquark and colored lepton pair production in $e^{+} e^{-}$ annihilations at TRISTAN, Phys. Lett. B 240 (1990) 243 [INSPIRE].

[37] CELLO collaboration, Search for Light Leptoquark Bosons, Phys. Lett. B 178 (1986) 452 [Addendum ibid. 184 (1987) 417] [INSPIRE].

[38] ALEPH collaboration, Searches for new particles in $Z$ decays using the ALEPH detector, Phys. Rept. 216 (1992) 253 [INSPIRE].

[39] DELPHI collaboration, Search for Leptoquarks and FCNC in $e^{+} e^{-}$annihilations at $\sqrt{s}=183 \mathrm{GeV}$, Phys. Lett. B 446 (1999) 62 [hep-ex/9903072] [INSPIRE].

[40] OPAL collaboration, Search for leptoquarks in electron photon scattering at $\sqrt{s_{e e}}$ up to $209 \mathrm{GeV}$ at LEP, Phys. Lett. B 526 (2002) 233 [hep-ex/0112024] [INSPIRE].

[41] OPAL collaboration, A Search for scalar leptoquarks in $Z^{0}$ decays, Phys. Lett. B 263 (1991) 123 [INSPIRE].

[42] OPAL collaboration, Search for pair produced leptoquarks in $e^{+} e^{-}$interactions at $\sqrt{s} \approx 189-209$ GeV, Eur. Phys. J. C 31 (2003) 281 [hep-ex/0305053] [INSPIRE].

[43] H1 collaboration, A Search for leptoquark bosons and lepton flavor violation in $e^{+} p$ collisions at HERA, Eur. Phys. J. C 11 (1999) 447 [Erratum ibid. 14 (2000) 553] [hep-ex/9907002] [INSPIRE].

[44] H1 collaboration, Search for Lepton Flavour Violation at HERA, Phys. Lett. B 701 (2011) 20 [arXiv:1103.4938] [INSPIRE].

[45] H1 collaboration, Search for first generation leptoquarks in ep collisions at HERA, Phys. Lett. B 704 (2011) 388 [arXiv:1107.3716] [INSPIRE].

[46] CDF collaboration, Search for third generation leptoquarks in $\bar{p} p$ collisions at $\sqrt{s}=1.8 \mathrm{TeV}$, Phys. Rev. Lett. 78 (1997) 2906 [INSPIRE].

[47] CDF collaboration, Search for first-generation scalar leptoquarks in $p \bar{p}$ collisions at $\sqrt{s}=1.96 \mathrm{TeV}$, Phys. Rev. D 72 (2005) 051107 [hep-ex/0506074] [InSPIRE]. 
[48] CDF collaboration, Search for second-generation scalar leptoquarks in $p \bar{p}$ collisions at $\sqrt{s}=1.96 \mathrm{TeV}$, Phys. Rev. D 73 (2006) 051102 [hep-ex/0512055] [INSPIRE].

[49] CDF collaboration, Search for Third Generation Vector Leptoquarks in p $\bar{p}$ Collisions at $\sqrt{s}=1.96 \mathrm{TeV}$, Phys. Rev. D 77 (2008) 091105 [arXiv:0706.2832] [INSPIRE].

[50] D0 collaboration, Search for third-generation leptoquarks in p $\bar{p}$ collisions at $\sqrt{s}=1.96 \mathrm{TeV}$, Phys. Rev. Lett. 99 (2007) 061801 [arXiv:0705.0812] [INSPIRE].

[51] D0 collaboration, Search for third generation scalar leptoquarks decaying into $\tau b$, Phys. Rev. Lett. 101 (2008) 241802 [arXiv:0806.3527] [INSPIRE].

[52] D0 collaboration, Search for pair production of second generation scalar leptoquarks, Phys. Lett. B 671 (2009) 224 [arXiv:0808.4023] [INSPIRE].

[53] D0 collaboration, Search for pair production of first-generation leptoquarks in p $\bar{p}$ collisions at $\sqrt{s}=1.96 \mathrm{TeV}$, Phys. Lett. B 681 (2009) 224 [arXiv:0907.1048] [INSPIRE].

[54] D0 collaboration, Search for Scalar Bottom Quarks and Third-Generation Leptoquarks in p $\bar{p}$ Collisions at $\sqrt{s}=1.96$ TeV, Phys. Lett. B 693 (2010) 95 [arXiv:1005.2222] [InSPIRE].

[55] ATLAS collaboration, Search for third generation scalar leptoquarks in pp collisions at $\sqrt{s}=7 \mathrm{TeV}$ with the ATLAS detector, JHEP 06 (2013) 033 [arXiv:1303.0526] [INSPIRE].

[56] ATLAS collaboration, Searches for third-generation scalar leptoquarks in $\sqrt{s}=13$ TeV pp collisions with the ATLAS detector, JHEP 06 (2019) 144 [arXiv: 1902.08103] [INSPIRE].

[57] ATLAS collaboration, Search for pairs of scalar leptoquarks decaying into quarks and electrons or muons in $\sqrt{s}=13 \mathrm{TeV}$ pp collisions with the ATLAS detector, JHEP 10 (2020) 112 [arXiv:2006.05872] [INSPIRE].

[58] ATLAS collaboration, Search for pair production of scalar leptoquarks decaying into first- or second-generation leptons and top quarks in proton-proton collisions at $\sqrt{s}=13$ TeV with the ATLAS detector, Eur. Phys. J. C 81 (2021) 313 [arXiv: 2010.02098] [INSPIRE].

[59] ATLAS collaboration, Search for pair production of third-generation scalar leptoquarks decaying into a top quark and a $\tau$-lepton in pp collisions at $\sqrt{s}=13 \mathrm{TeV}$ with the ATLAS detector, JHEP 06 (2021) 179 [arXiv:2101.11582] [INSPIRE].

[60] CMS collaboration, Search for pair production of third-generation scalar leptoquarks and top squarks in proton-proton collisions at $\sqrt{s}=8 \mathrm{TeV}$, Phys. Lett. B 739 (2014) 229 [arXiv: 1408.0806] [INSPIRE].

[61] CMS collaboration, Search for single production of scalar leptoquarks in proton-proton collisions at $\sqrt{s}=8 \mathrm{TeV}$, Phys. Rev. D 93 (2016) 032005 [Erratum ibid. 95 (2017) 039906] [arXiv: 1509.03750] [INSPIRE].

[62] CMS collaboration, Search for third-generation scalar leptoquarks and heavy right-handed neutrinos in final states with two tau leptons and two jets in proton-proton collisions at $\sqrt{s}=13 \mathrm{TeV}, \mathrm{JHEP} 07$ (2017) 121 [arXiv: 1703.03995] [INSPIRE].

[63] CMS collaboration, Search for a singly produced third-generation scalar leptoquark decaying to a $\tau$ lepton and a bottom quark in proton-proton collisions at $\sqrt{s}=13 \mathrm{TeV}$, JHEP 07 (2018) 115 [arXiv: 1806.03472] [INSPIRE].

[64] CMS collaboration, Search for leptoquarks coupled to third-generation quarks in proton-proton collisions at $\sqrt{s}=13$ TeV, Phys. Rev. Lett. 121 (2018) 241802 [arXiv: 1809.05558] [INSPIRE]. 
[65] CMS collaboration, Search for heavy neutrinos and third-generation leptoquarks in hadronic states of two $\tau$ leptons and two jets in proton-proton collisions at $\sqrt{s}=13 \mathrm{TeV}, \mathrm{JHEP} \mathbf{0 3}$ (2019) 170 [arXiv: 1811.00806] [INSPIRE].

[66] M. Leurer, A Comprehensive study of leptoquark bounds, Phys. Rev. D 49 (1994) 333 [hep-ph/9309266] [INSPIRE].

[67] S. Davidson, D.C. Bailey and B.A. Campbell, Model independent constraints on leptoquarks from rare processes, Z. Phys. C 61 (1994) 613 [hep-ph/9309310] [INSPIRE].

[68] E. Gabrielli, Model independent constraints on leptoquarks from rare $\mu$ and $\tau$ lepton processes, Phys. Rev. D 62 (2000) 055009 [hep-ph/9911539] [InSPIRE].

[69] R. Mandal and A. Pich, Constraints on scalar leptoquarks from lepton and kaon physics, JHEP 12 (2019) 089 [arXiv:1908.11155] [INSPIRE].

[70] SINDRUM II collaboration, Improved limit on the branching ratio of $\mu^{-} \rightarrow e^{+}$conversion on titanium, Phys. Lett. B 422 (1998) 334 [InSPIRE].

[71] A.J. Baltz et al., The Physics of Ultraperipheral Collisions at the LHC, Phys. Rept. 458 (2008) 1 [arXiv: 0706.3356] [INSPIRE].

[72] S.R. Klein and P. Steinberg, Photonuclear and Two-photon Interactions at High-Energy Nuclear Colliders, Ann. Rev. Nucl. Part. Sci. 70 (2020) 323 [arXiv:2005. 01872] [inSPIRE].

[73] J. Alwall et al., The automated computation of tree-level and next-to-leading order differential cross sections, and their matching to parton shower simulations, JHEP 07 (2014) 079 [arXiv: 1405.0301] [INSPIRE].

[74] T. Sjöstrand, S. Mrenna and P.Z. Skands, PYTHIA 6.4 Physics and Manual, JHEP 05 (2006) 026 [hep-ph/0603175] [INSPIRE].

[75] DELPHES 3 collaboration, DELPHES 3, A modular framework for fast simulation of a generic collider experiment, JHEP 02 (2014) 057 [arXiv: 1307.6346] [INSPIRE].

[76] Leptoquark toolbox, https://lqnlo.hepforge.org/.

[77] I. Doršner and A. Greljo, Leptoquark toolbox for precision collider studies, JHEP 05 (2018) 126 [arXiv: 1801.07641 ] [INSPIRE].

[78] ATLAS collaboration, Measurement of exclusive $\gamma \gamma \rightarrow W^{+} W^{-}$production and search for exclusive Higgs boson production in pp collisions at $\sqrt{s}=8 \mathrm{TeV}$ using the ATLAS detector, Phys. Rev. D 94 (2016) 032011 [arXiv:1607.03745] [InSPIRE].

[79] ATLAS collaboration, Observation of photon-induced $W^{+} W^{-}$production in pp collisions at $\sqrt{s}=13 \mathrm{TeV}$ using the ATLAS detector, CERN-EP-2020-165 (2020) [Phys. Lett. B 816 (2021) 136190] [arXiv: 2010.04019] [INSPIRE].

[80] S. White, The ATLAS zero degree calorimeter, Nucl. Instrum. Meth. A 617 (2010) 126 [INSPIRE].

[81] ATLAS collaboration, Exclusive dimuon production in ultraperipheral $\mathrm{Pb}+\mathrm{Pb}$ collisions at $\sqrt{s_{\mathrm{NN}}}=5.02 \mathrm{TeV}$ with $A T L A S$, arXiv:2011.12211 [INSPIRE].

[82] A. Elagin, P. Murat, A. Pranko and A. Safonov, A New Mass Reconstruction Technique for Resonances Decaying to $\tau \tau$, Nucl. Instrum. Meth. A 654 (2011) 481 [arXiv:1012.4686] [INSPIRE].

[83] L.-G. Xia, A mass reconstruction technique for a heavy resonance decaying to $\tau^{+} \tau^{-}$, Chin. Phys. C 40 (2016) 113003 [arXiv:1601.02454] [INSPIRE]. 
[84] G. Cowan, K. Cranmer, E. Gross and O. Vitells, Asymptotic formulae for likelihood-based tests of new physics, Eur. Phys. J. C 71 (2011) 1554 [Erratum ibid. 73 (2013) 2501] [arXiv: 1007.1727] [INSPIRE].

[85] A. Hocker et al., TMVA - Toolkit for Multivariate Data Analysis, physics/0703039 [INSPIRE].

[86] L.-G. Xia, Understanding the boosted decision tree methods with the weak-learner approximation, arXiv:1811.04822 [INSPIRE].

[87] ATLAS collaboration, Observation of light-by-light scattering in ultraperipheral $\mathrm{Pb}+\mathrm{Pb}$ collisions with the ATLAS detector, Phys. Rev. Lett. 123 (2019) 052001 [arXiv:1904.03536] [INSPIRE].

[88] ATLAS collaboration, Luminosity determination in pp collisions at $\sqrt{s}=8 \mathrm{TeV}$ using the ATLAS detector at the LHC, Eur. Phys. J. C 76 (2016) 653 [arXiv:1608. 03953] [inSPIRE].

[89] ATLAS collaboration, Electron efficiency measurements with the ATLAS detector using the 2015 LHC proton-proton collision data, ATLAS-CONF-2016-024 (2016).

[90] ATLAS collaboration, Muon reconstruction performance of the ATLAS detector in proton-proton collision data at $\sqrt{s}=13 \mathrm{TeV}$, Eur. Phys. J. C $\mathbf{7 6}$ (2016) 292 [arXiv: 1603. 05598] [INSPIRE].

[91] ATLAS collaboration, Measurement of the tau lepton reconstruction and identification performance in the ATLAS experiment using pp collisions at $\sqrt{s}=13 \mathrm{TeV}$, ATLAS-CONF-2017-029 (2017).

[92] ATLAS collaboration, Performance of missing transverse momentum reconstruction with the ATLAS detector using proton-proton collisions at $\sqrt{s}=13$ TeV, Eur. Phys. J. C 78 (2018) 903 [arXiv: 1802.08168] [INSPIRE].

[93] ATLAS collaboration, Jet reconstruction and performance using particle flow with the ATLAS Detector, Eur. Phys. J. C 77 (2017) 466 [arXiv:1703.10485] [InSPIRE].

[94] ATLAS collaboration, Jet energy scale and resolution measured in proton-proton collisions at $\sqrt{s}=13 \mathrm{TeV}$ with the ATLAS detector, arXiv:2007.02645 [INSPIRE].

[95] ATLAS Experiments, https://atlas.web.cern.ch/Atlas/GROUPS/PHYSICS/PAPERS/EXOT-2017-30/. 